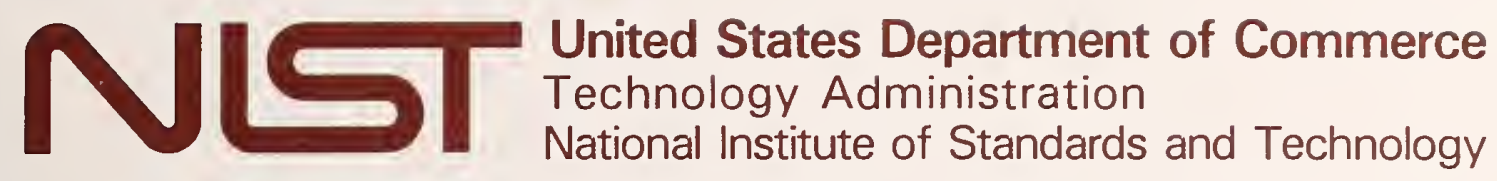

NIST Technical Note 1509

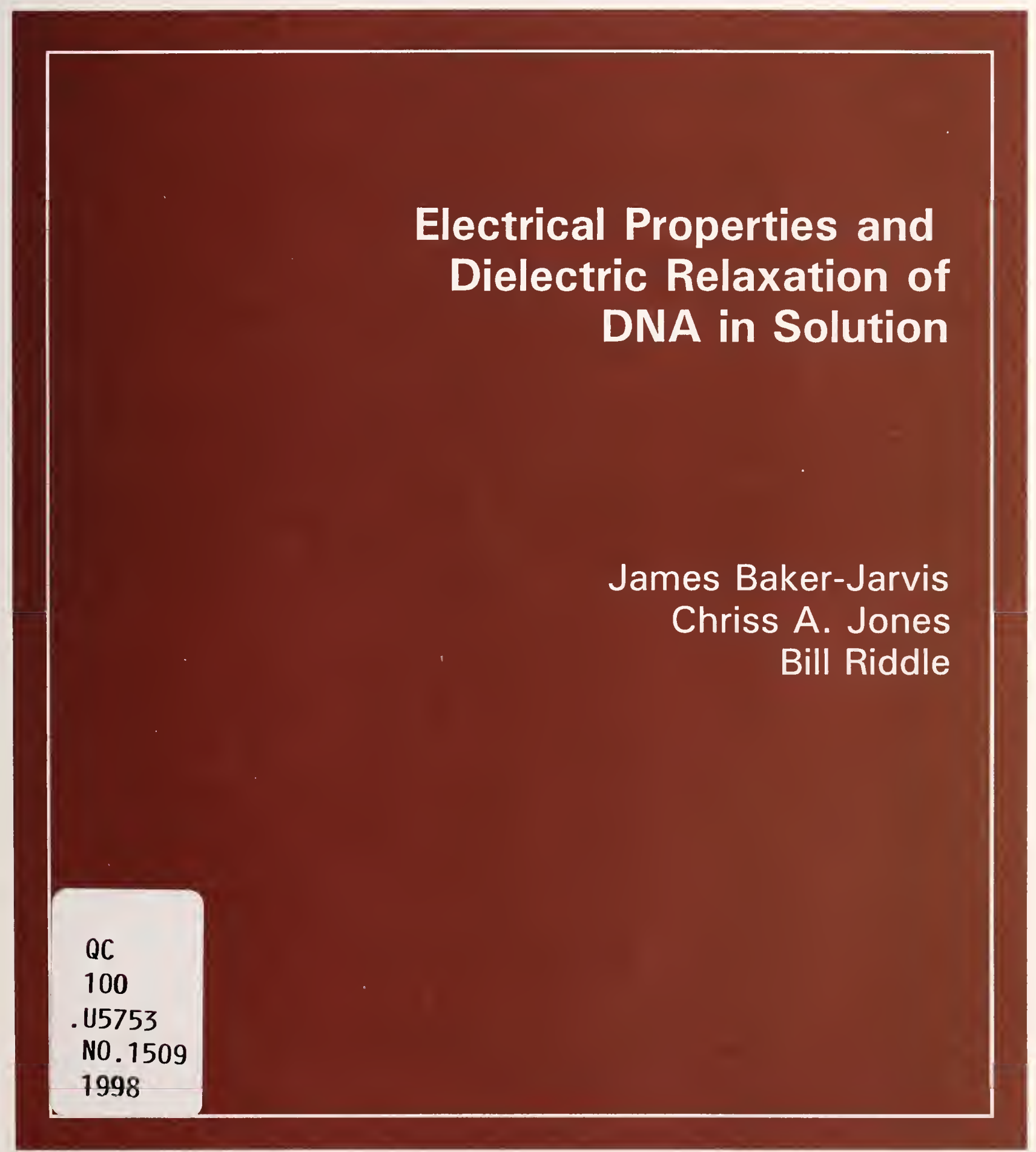



NIST Technical Note 1509

\section{Electrical Properties and Dielectric Relaxation of DNA in Solution}

James Baker-Jarvis

Chriss A. Jones

Bill Riddle

Radio-Frequency Technology Division

Electronics and Electrical Engineering Laboratory

National Institute of Standards and Technology

325 Broadway

Boulder, Colorado 80303-3328

November 1998

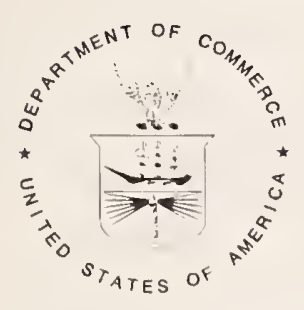

U.S. DEPARTMENT OF COMMERCE, William M. Daley, Secretary TECHNOLOGY ADMINISTRATION, Gary R. Bachula, Acting Under Secretary for Technology NATIONAL INSTITUTE OF STANDARDS AND TECHNOLOGY, Raymond G. Kammer, Director 
National Institute of Standards and Technology Technical Note

Natl. Inst. Stand. Technol., Tech. Note 1509, 72 pages (November 1998) CODEN:NTNOEF

U.S. GOVERNMENT PRINTING OFFICE

WASHINGTON: 1998

For sale by the Superintendent of Documents, U.S. Government Printing Office, Washington, DC 20402-9325 


\section{Contents}

1 Introduction 2

2 Amino Acids, Peptides, and Proteins: An Overview 7

3 Structure and Electrical Properties of DNA 10

3.1 Charges and Electrical Properties of the DNA Molecule . . . . . . . 10

3.2 Dielectric Properties of Bound Water and Polyelectrolytes Around DNA 13

3.3 Response of DNA in Electric Driving Fields . . . . . . . . . . . 14

3.4 Dynamics of Polarization Relaxation . . . . . . . . . . . . 17

3.5 The Electric Field near the DNA Molecule . . . . . . . . . . . . 19

4 Counterions and the Helix $\quad 19$

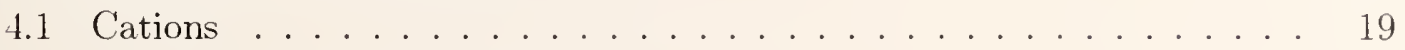

4.2 Helix-Coil Transition in DNA . . . . . . . . . . . . 23

5 Modeling the Potential around the DNA Molecule 26

5.1 Phenomenological Models of DNA Dynamics . . . . . . . . . . 26

5.2 Poisson-Boltzmann Equation . . . . . . . . . . . 27

5.3 Poisson-Boltzmann Applied to Modeling the DNA Helix . . . . . . . . 29

5.4 Numerical Models for DNA Dynamics . . . . . . . . . . . . . 30

6 Phenomenological Models of Dipole Moment, Relaxation Time, and Polarizability 31

6.1 Persistence Length and Radius of Gyration . . . . . . . . . . . . . . 31

6.2 Polarization . . . . . . . . . . . . . . . . . . . 31

6.3 Simple Model of Dipole Moment . . . . . . . . . . . . . . . . 33

6.4 Dipole Moment, Permittivity, and Relaxation Time . . . . . . . . . . 35

6.5 Statistical-Mechanical Model of Polarization for Rod-Like DNA . . . . 36

7 Charge Transfer in DNA 39

8 Measurements of DNA $\quad 42$

8.1 Overview of Past Measurements . . . . . . . . . . . . . . . 42

8.2 Permittivity Measurements and Electrode Polarization ......... 43 
8.3 Measurement Methods . . . . . . . . . . . . . . . . . . 47

8.3.1 Capacitive Measurement Techniques . . . . . . . . . . . . . . 47

8.3 .2 Four-Probe Technique . . . . . . . . . . . . 47

8.3.3 The Open-Circuited Holder for Liquid Measurement . . . . . . . 48

8.4 Dielectric Measurements on DNA in Solution . . . . . . . . . . 49

9 Discussion and Conclusions $\quad 58$

10 References $\quad 59$ 


\title{
Electrical Properties and Dielectric Relaxation of DNA in Solution
}

\author{
James Baker-Jarvis* $\quad$ Chriss A. Jones * Bill Riddle *
}

November 20, 1998

In this report we overview and summarize the electrical properties and dielectric relaxation of solvenated DNA. We review models for counterion dynamics, dipole moment, polarizability, and relaxation in both double- and single-stranded DNA. We also present dielectric measurements on double- and single-stranded DNA from herring sperm in protamine solutions. The analysis is based on polymer dynamics and statistical mechanics. The observed dipole moment of DNA is induced through the distortion of the counterion atmosphere by the applied electric field. Doublestranded DNA does not possess a permanent dipole moment except in the case where ligands are attached. We find evidence that denaturation proceeds in stages. The first stage is when counterions are expelled from the molecule which causes dissociation through strong phosphate-phosphate repulsion between strands. This increases the conductivity of the solution. We also analyze dispersion of DNA solutions from low to microwave frequencies. We found evidence of low-frequency dispersion which is consistent with counterion-sheath relaxation models. We also found a dispersion at megahertz frequencies which is consistent with site-bound counterion models.

Key words: counterions; dielectric constant: dipole monent; DNA: liquids: loss factor; microwave measurements; permittivity measurement: protamine: relaxation: single-stranded DNA.

"Radio-Frequency Technology Division, MS \$13.01, National Inst itute of Standards and Technology: Boulder, CO $80303-3328$ email:jjarris @boulder.nist.gov 


\section{Introduction}

This report examines the dielectric relaxation and electrical properties of deoxyribonucleic acid (DNA) in alternating electric fields $[1,2]$.

The literature on dielectric relaxation of DNA is copious [1-16]. There have been a number of reviews of the polyelectrolyte properties of DNA [10-14], but none on electrical properties and dielectric relaxation of DNA. [3-5]

Nucleic acids are high-molecular mass polymers formed of pyrimidine and purine bases, a sugar, and phosphoric-acid backbone as shown in figure 1.1. Nucleic acids are built up of nucleotide units which are composed of sugar, base, and phosphate groups in helical conformation. Nucleotides are linked by three phosphates groups which are designated $\alpha, \beta$, and $\gamma$. The phosphate groups are linked through the pyrophosphate bond. The individual nucleotides are joined together by groups of phosphates that form the phosphodiester bond between the $3^{\prime}$ and $5^{\prime}$ carbon atoms of successive sugars (see figure 1.2). These phosphate groups are acidic. Polynucleotides have a hydroxyl group at one end and a phosphate group on the other end. Nucleosides are subunits of nucleotides and contain a base and a sugar. The bond between the sugar and base is called the glycosidic bond as indicated in figure 1.3. The base can rotate only in sterically permissible orientations about the glycosidic bond.

The helix is formed from two strands. The bases in adjacent strands combine by hydrogen bonding, an electrostatic interaction, with a pyrimidine on one side and purine on the other. In DNA the purine adenine (A) pairs with the pyrimidine thymine (T). The purine guanine $(\mathrm{G})$ pairs with the pyrimidine cytosine $(\mathrm{C})$. These are shown in figures 1.2 and 1.3. A hydrogen bond is formed between a covalently bonded donor hydrogen atom that is positively charged and a negatively charged acceptor atom. The A-T base pair associates by two hydrogen bonds, whereas C-G base pairs associate by three hydrogen bonds. The base-pair sequence is the carrier of genetic information. The genetic code is formed of a sequence of three base pairs which determines a type of amino acid. For example, the sequence of $\underbrace{T T T}_{\text {phe }} \underbrace{A A A}_{\text {lyso }} \underbrace{A A G}_{\text {lys }} \underbrace{G C T}_{\text {ala }}$ determines an amino acid sequence of: phenylalanine-lysine-lysine-alanine.

The DNA molecule has a net negative charge due to the phosphate backbone. When dissolved in a cation solution, some of the charge of the molecule is neutralized by cations. It is generally thought that the double-stranded DNA molecule has little in- 


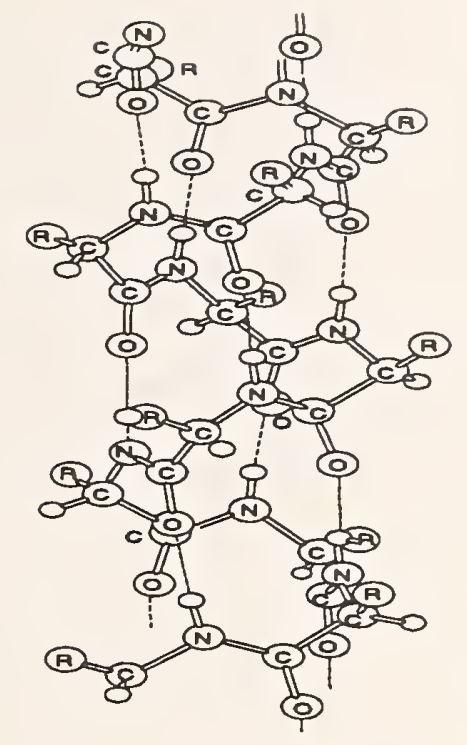

Figure 1.1. The right-handed helical conformation of DNA.

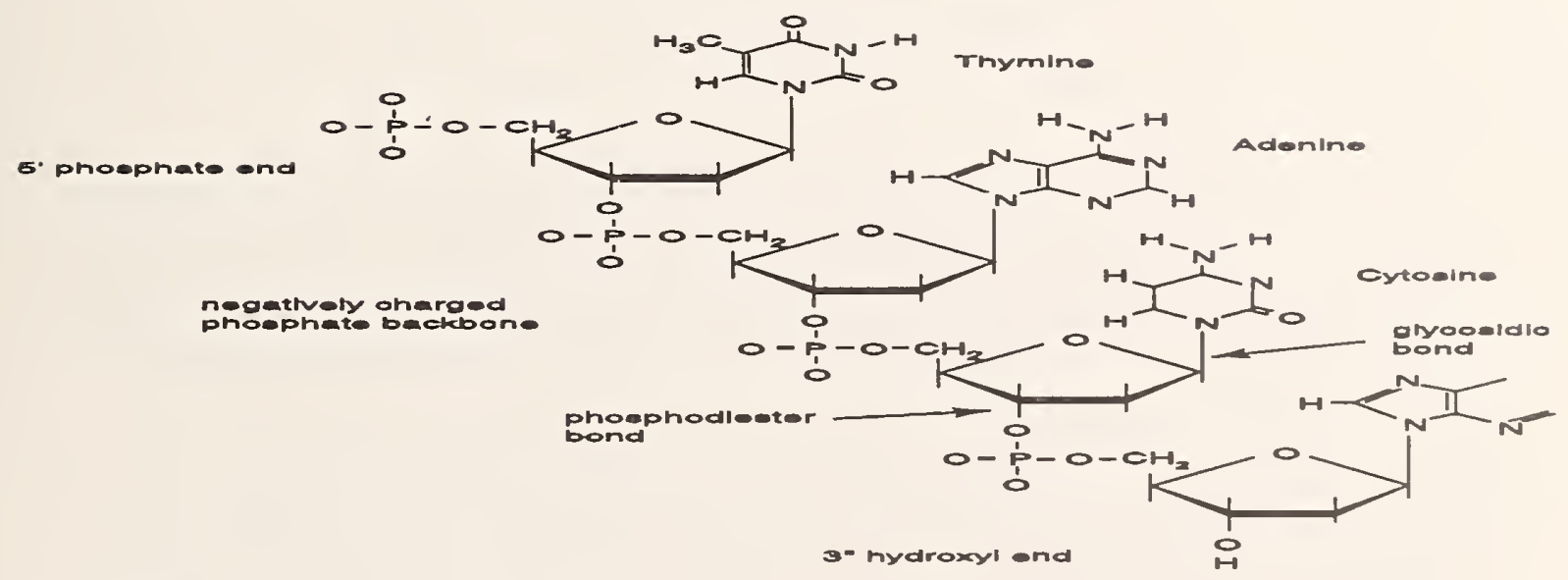

Figure 1.2. Single strand of DNA and the phosphodiester and glycosidic bonds. 


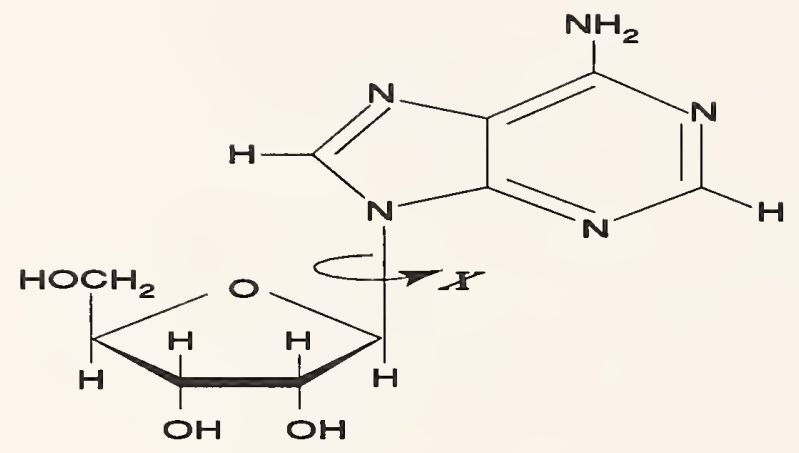

Figure 1.3. Glycosidic bond denoted by X.

trinsic permanent dipole moment (see figure 1.4). This is because the two strands that compose the helix are oriented so the dipole moment of one strand cancels the other. However, when DNA is dissolved in a solvent, such as saline solution, an induced dipole moment forms due to reorganization of charge into a layer around the molecule called the counterion sheath.

Watson and Crick concluded through X-ray diffraction studies that the structure of DNA is in the form of a double-stranded helix. In addition to x-ray structure experiments on DNA, information has been gleaned through nuclear magnetic resonance (NMR) experiments. Types $\mathrm{A}$ and B DNA are in the form of right-handed helices. Type $\mathrm{Z}$ DNA is in a left-handed conformation. There is a Type $\mathrm{B}$ to $\mathrm{Z}$ transition between conformations. A transition from Type A to Type B DNA occurs when DNA is dissolved in a solvent [6]. The Watson-Crick conception of DNA as a uniform helix is an approximation. In reality DNA exists in many conformations and may contain inhomogeneities such as attached proteins. In general, double-stranded DNA is not a rigid rod, but rather a meandering worm-like chain. Once formed, even though the individual bonds composing DNA are weak, the molecule as a whole is very stable. The helical 

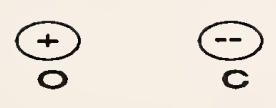

(a)

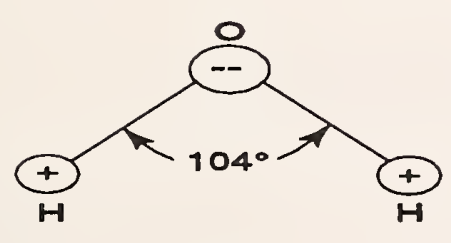

(c)

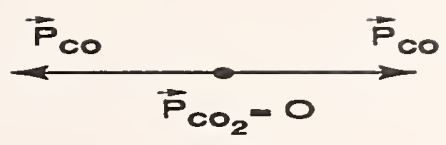

(b)

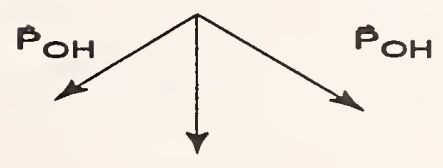

$\overrightarrow{\mathrm{P}}_{\mathrm{H}_{2} \mathrm{O}}$

(d)

Figure 1.4. Permanent dipole moments of molecules. In (a) the charge in the carbon dioxide molecule is depicted. (b) depicts the cancelation of the dipole moments in carbon dioxide. (c) and (d) describe the dipole moment of the water molecule. 


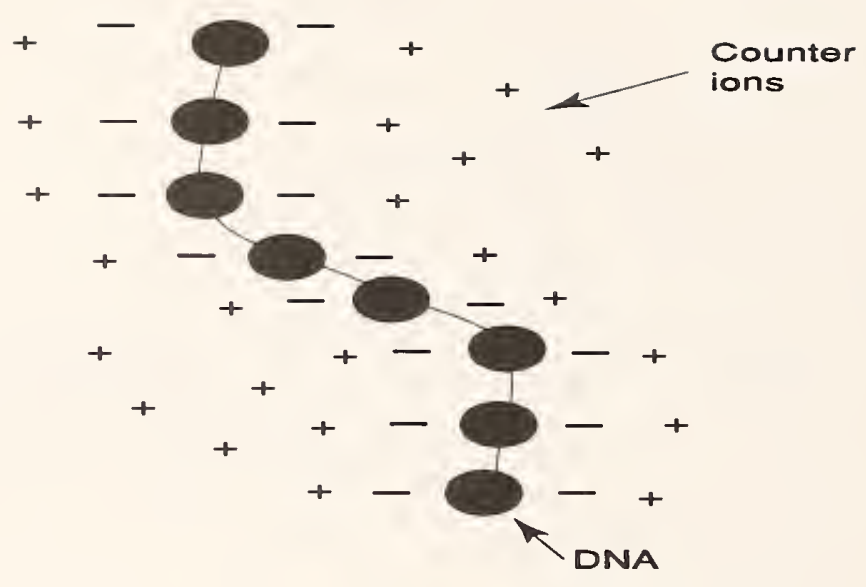

Figure 1.5. Counterions and DNA molecule.

form of the DNA molecule produces major and minor grooves in the outer surface of the molecule. There are also bound-water molecules in the grooves. Many interactions between proteins or protons with DNA occur in these grooves. Sections 2 and 3 present an overview of the charge structure and dipole properties of DNA.

The interaction of the counterions with the DNA molecule has been a subject of intensive research over the years. Some of the counterions bind to the phosphate backbone with a weak covalent bond. Other counterions are more loosely bound and some may penetrate into the major and minor grooves $[7,8]$. It is assumed that ions become bound near charges in the DNA molecule and a double layer forms. The ions attracted to the charged DNA molecule forms a counterion sheath that shields some of the charge of the DNA. This is shown in figure 1.5. The counterion sheath around a DNA molecule is composed of cations such as $\mathrm{Na}$ or $\mathrm{Mg}$, which are attracted to the backbone negative phosphate charges as shown in figure 1.5. These charges are somewhat mobile and oscillate about phosphate charge centers in an applied electric field. A portion of these counterions is condensed near the surface of the molecule, whereas the vast majority are diffusely bound. The condensed counterions bind with the phosphate backbone 
charges in a weakly covalent bond. In this bond, the outer s-shell of the counterion may hybridize with the s and p shells of the phosphate oxygen [9]. Sakamoto [10] also studied dielectric relaxation of DNA in ethanol instead of saline solution. This study lends further proof to the counterion-dipole theory of relaxation.

The current understanding of the counterion atmosphere around the helix and the helix-coil transition is overviewed in Section 4.

An overview of relaxation time, polarization, and a statistical-mechanical theory of relaxation is presented in Section 6. Section 7 presents an overview of charge transfer. Section 8 discusses dielectric measurements and summarizes our measurement results on both denatured and double-stranded DNA in protamine solutions over a frequency range of $20 \mathrm{~Hz}$ to $200 \mathrm{MHz}$.

\section{Amino Acids, Peptides, and Proteins: An Overview}

In this section we will overview dielectric relaxation of the building blocks of proteins, amino acids, and peptides (see figure 2.1). Amino acids contain carboxyl (COOH) groups, amide $\left(\mathrm{NH}_{2}\right)$ groups, and side groups $(\mathrm{R})$, as shown in figure 2.1. The side groups and the dipole moment of the amino and carboxyl groups determine most of the dielectric properties of the acid. Some of the side groups are polar; others are nonpolar. When ionized, the amino and carboxyl groups have positive and negative charges respectively. This charge separation forms a permanent dipole. $\alpha$ amino acids have an amino group and carboxyl group on the same carbon denoted $C_{\alpha} \cdot \alpha$-amino acids have a dipole moment of 15 to 17 debyes (D) ( 1 debye equals $3.33 \times 10^{-30}$ coulombmeter). $\beta$ amino acids have a $\mathrm{CH}_{2}$ group in between the amino and carboxyl groups which produces a large charge separation and therefore a dipole moment on the order of $20 \mathrm{D}$.

Peptides are formed from condensed amino acids. A peptide consists of a small number of amino acids connected by peptide bonds. Peptide bonds (see figure 2.2) provide linkages to amino acids through the $\mathrm{CO}-\mathrm{NH}$ bond by using a water molecule as a bridge (see figure 3.1). The peptide unit has a dipole moment as shown in figure 2.2 on the order of $3.7 \mathrm{D}$. Chains of amino acids are called polyamino acids or polypeptides. These are terminated by an amide group on one end and a carboxyl group on the other. Typical dipole moments for polypeptides are on the order of $1000 \mathrm{D}$. 


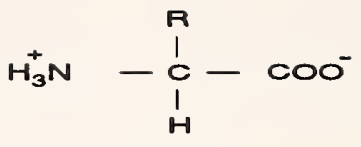

$$
\begin{aligned}
& \mathrm{H}_{3}^{+} \mathrm{N}-\stackrel{\substack{H \\
1 \\
H}}{\mathrm{H}}-\mathrm{COO}^{-}
\end{aligned}
$$

Figure 2.1. Amino acids: (a) standard form for amino acid where $\mathrm{R}$ is a side chain, (b) glycine, and (c) valine.

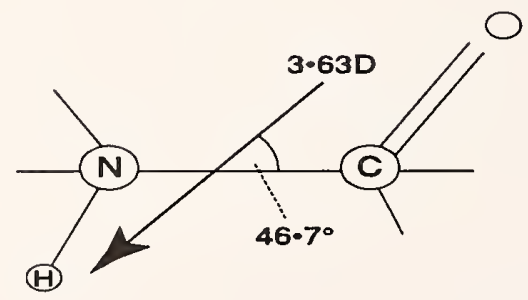

Figure 2.2. Peptide bonds and dipole moment. 


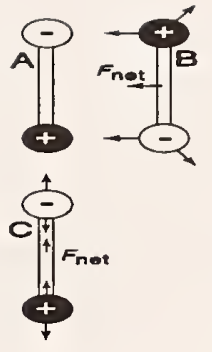

(a)

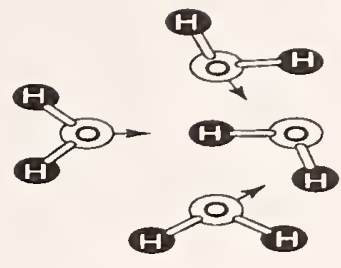

(b)

Figure 2.3. Origins of hydrogen and van der Waals bonds. In (a) the attractive force between dipoles is depicted. In (b) the attractive force between water molecules due to charge separation is shown.

Polyamino acids can be either in the helical or random-coil phase. In the helical state, $\mathrm{C}=\mathrm{O}$ bonds are linked by hydrogen bonds (see figure 2.3 ) to NH groups. The helix can either be right-handed or left-handed; however, the right-handed helix is more stable. Generally, polyamino acids have permanent dipole moments and dielectric relaxation frequencies in the kilohertz region. The dipole moment of polyamino acids has been found to satisfy [11]

$$
<\mu^{2}>=N_{0} M_{p} \frac{9 k_{B} T}{4 \pi P},
$$

where $M_{p}$ is molecular mass of the protein, $k_{B}$ is Boltzmann's constant, $T$ is temperature, $P$ is the number of residues, and $N_{0}$ is a constant. The brackets $\langle>$ on the left side of eq (2.1) denote ensemble average.

The origin of relaxation in proteins has been debated over the years. We know that proteins are composed of polyamino acids with permanent dipole moments, but they also have free and loosely bound protons. These protons bind loosely to the carboxyl and amino groups. It has been hypothesized that much of observed relaxation behavior of proteins is due to movement of these nearly free protons in the applied field [12) Strong protonic conductivity has also been observed in DNA. At present, the consensus is that both permanent dipoles and the proton-induced polarization contribute to dielectric 
relaxation of proteins.

There are three distinct dielectric relaxations in proteins [1]. The first is in the 10 $\mathrm{kHz}$ to $1 \mathrm{MHz}$ region and is due to rotation of the protein side chains. The second relaxation occurs from $100 \mathrm{MHz}$ to $10 \mathrm{GHz}$ and is thought to be due to bound water. The third relaxation is around $10 \mathrm{GHz}$ and is due to free water. Bound water relaxes at a frequency lower than free water due to the binding to other atoms. The relaxation frequency in gigahertz of bound water depends on binding energy $\Delta E$ as [13]

$$
f_{B}=18 \exp (-\Delta E / R T)
$$

Nandi [14] developed a theory using correlation functions that adequately predicts dispersion in aqueous protein solutions. This theory also predicts an increase in the static permittivity as the concentration of protein increases.

The mean-squared dipole moment of proteins has been modeled as linear in molecular mass $M_{p}$ and has the form [11]

$$
<\mu^{2}>=\frac{9000 k_{B} T \Delta \epsilon_{r} M_{p}}{4 \pi P h} .
$$

Here $\Delta \epsilon_{r}$ is the dielectric increment, $h$ is an empirical parameter, and $N$ is the number of residues. In presenting dielectric measurements frequently the difference between the real part of the permittivity at a given frequency minus $\epsilon_{\infty}, \Delta \epsilon_{r}=\epsilon_{r}-\epsilon_{\infty}$, is called the dielectric increment. $\epsilon_{\infty}$ is obtained from the Cole-Cole plot. The value of the dielectric increment varies from molecule to molecule. There are various other definitions of dielectric increment used in the literature such as specific dielectric increment which normalized to the concentration. The dielectric increment depends on cation and cation concentration. Typical dipole moments of proteins are on the order of $500 \mathrm{D}$. In the $\alpha$-helix conformation the amide groups are aligned parallel to the axis of the helix. This produces a repulsive force between successive dipoles. In long $\alpha$ helices, dipole-dipole interactions between the amide groups can be appreciable.

\section{Structure and Electrical Properties of DNA}

\subsection{Charges and Electrical Properties of the DNA Molecule}

Dimensional information for the DNA molecule is given in table 3.1. The dominant charges on the DNA molecule are from oxygen atoms in the phosphate groups located 
on the outer surfaces of the helix. The DNA molecule also has additional 45 partially charged atoms per base-pair site. The charge on DNA has been studied by Renugopalakrishnan and Lakshminarayanan [15] and by Pearlman.

Table 3.1. Dimensions in DNA.

\begin{tabular}{cc}
\hline Location & Typical lengths (nm) \\
\hline Radius of Type A DNA & 0.9 \\
Base-pair vertical separation & 0.34 \\
Intrastrand phosphate charge separation & 0.70 \\
counterion sheath thickness & 0.30 \\
\hline
\end{tabular}

Table 3.2. Typical bond energies.

\begin{tabular}{cc}
\hline Bond Type & Typical binding energy $(\mathrm{J} / \mathrm{mol})$ \\
\hline Covalent & $-2.8 \times 10^{5}$ \\
Ionic & $-1.8 \times 10^{5}$ \\
Hydrogen & $-1.8 \times 10^{4}$ \\
Van der Waals & $-2.8 \times 10^{3}$ \\
\hline
\end{tabular}

Bases are stacked vertically by van der Waals forces, which originate in the attraction of induced dipole moments between atoms as shown in figure 2.3. Hydrogen bonding between bases originates from the attractive force between dipole moments and requires the dissociation of a water molecule (see figures 1.4 and 2.3). To illustrate the relative strength of the hydrogen bond, typical strengths of bond types are given in table 3.2. The helix strands can be either separated by heating the DNA solute followed by quenching or by decreasing the ionic strength of the solvent. The strands also unwind by enzymic interactions with ribonucleic acid (RNA) for self replication. The reaction of strand separation is called melting denaturation. The modeling of the helix-coil transition requires a detailed analysis of polyelectrolyte theory coupled with free-energy considerations. If the strands separate, they generally will coil. Single-stranded DNA in a rigid, oriented state has a permanent dipole moment due to the alignment of the 
positively charged amide groups. Single-stranded DNA has a permanent dipole moment of approximately $20 \mathrm{D}$ per base and bases are separated by $0.34 \mathrm{~nm}$. However, coiling severely decreases the net permanent dipole moment [16].

Double-stranded DNA possesses a large induced dipole moment due to the counterion atmosphere, on the order of thousands of debye. This fact is gleaned from both dielectric relaxation studies, birefringence and dichroism experiments $[17]$, and other light-scattering experiments [18]. The induced dipole moment $\vec{\mu}$ in an electric field $\vec{E}$ is defined in terms of the polarizability $\vec{\mu}=\alpha \cdot \vec{E}$. The polarizability $\alpha$ has been calculated from statistical-mechanical means in terms of the mean-squared dipole moment $\left\langle\mu^{2}\right\rangle$ as $[19]$

$$
\alpha=\frac{d<\mu>_{E=0}}{d E}=\frac{<\mu^{2}>_{E=0}}{k_{B} T},
$$

where $T$ is the temperature and $k_{B}$ is Boltzmann's constant. The right-hand side of eq. (3.1) shows that polarizability is a result of fluctuations in the dipole moment.

Since the individual strands of double-stranded DNA are antiparallel and the molecule is symmetrical, the transverse dipole moments should cancel. However, a number of researchers have measured a small permanent dipole moment for DNA [20]. In alternating fields, the symmetry of the molecule may be deformed slightly to produce a small permanent dipole moment [1]. Another origin of the apparent, small permanent dipole moment is attached charged ligands such as proteins or multivalent cations [21]. These ligands produce a net dipole moment on the DNA molecule by breaking the symmetry. The question of how much of the relaxation of the DNA molecule is due to induced dipole moment versus permanent moment has been studied by Hogan [17].

The response of permanent versus induced dipole moment differs in terms of field strength. The potential energy of a permanent dipole moment at an angle $\theta$ to the electric field is $U=-\mu E \cos \theta$, whereas the induced dipole moment in the electric field is quadratic, $U=-(\Delta \alpha / 2) E^{2} \cos ^{2} \theta$, where $\Delta \alpha$ is the difference in polarizability along anisotropy axes of the molecule. Experiments indicate that the majority of the moment was induced rather than permanent.

Crothers have studied the effects of charge imbalance in DNA strands which they have linked to DNA bending. This could happen for single-stranded DNA since $[22,23]$ DNA bends when attached proteins neutralize one side of the DNA molecule. Repulsions between negative phosphate charges in the rest of the helix cause bending. This bending 
is postulated to cause a permanent dipole moment since the phosphate charge balance is changed $[17,21,24]$.

\subsection{Dielectric Properties of Bound Water and Polyelectrolytes Around DNA}

The region close to the DNA molecule has a low dielectric constant and fixed charge. The region far from the molecule has a dielectric constant close to that of water. Lamm [25] studied the variation of dielectric constant in the grooves, near the surface, and far away from the DNA molecule. Table 3.3 lists predictions for dielectric constants in various regions around the molecule. The permittivity depends on solvent concentration, distance from the molecule, boundary effects, and dielectric field-saturation. The variation of dielectric constant with position significantly alters the predictions for the electric potential in the groove regions. Model predictions depend crucially on knowing the dielectric constant of water. Numerical modeling of the DNA molecule depends critically on the dielectric constant of water. When a dielectric constant of water that varies in space is used, numerical models indicate that small ions such as hydrogen can penetrate into the minor and major grooves $[8,26]$. These predictions are not obtained for models using spatially-independent dielectric constants for water.

The molecular structure of water is not simple. Besides the basic $\mathrm{H}_{2} \mathrm{O}$ triad structure of the water molecule (see figure 1.4), there are also complicated hydrogen-bonded networks created by dipole-dipole interactions which form hydroxyl $\left(\mathrm{OH}^{-}\right)$and hydronium $\left(\mathrm{H}_{3} \mathrm{O}^{+}\right)$ions. The dielectric constant of water is about 80 , whereas biological water contains ions which affect both the real and imaginary parts of the permittivity. Water bound in proteins and DNA has a decreased permittivity. This is due to constraints on the movement of the molecules when they are attached to biomaterials.

DNA contains bound-water molecules. There are estimated to be 5 to 20 boundwater molecules per base pair [13]. Some of the bound water in the minor and major grooves of DNA located on the charged nitrogenous bases and forms an aqueous bridge or water backbone [13] (see figure 3.1). This water is tightly bound and rotational motion is constrained in response to oscillating electric fields and therefore has a lower dielectric constant. Water molecules are also bound to the negatively charged phosphate groups on the exterior of the helix. In Type-B DNA the phosphate groups are far apart and the water molecules are arranged independently around the phosphates. In TypeA DNA the water molecules form a continuous water bridge between the phosphate 
Table 3.3. Real part of the permittivity around the DNA molecule as a function of distance in nanometers from the center of the molecule and as a function of solvent concentration in moles per liter $(\mathrm{mol} / \mathrm{l})$.

\begin{tabular}{cccc}
\hline radius $(\mathrm{nm})$ & Location & $\epsilon(0 \mathrm{~mol} / \mathrm{l})$ & $\epsilon(0.5 \mathrm{~mol} / \mathrm{l})$ \\
\hline \hline 0.07 & major groove & 20 & 20 \\
0.07 & minor groove & 46 & 45 \\
0.07 & elsewhere & 23 & 23 \\
\hline 0.21 & major groove & 31 & 27 \\
0.21 & minor groove & 29 & 26 \\
0.21 & elsewhere & 34 & 30 \\
\hline 0.35 & major groove & 59 & 56 \\
0.35 & minor groove & 41 & 38 \\
0.35 & elsewhere & 55 & 52 \\
\hline 1.3 & away from molecule & 77 & 73 \\
\hline
\end{tabular}

sites. In Type-Z DNA, water molecules form bridges between amino groups in bases and oxygens in the phosphate groups $[13,27]$.

\subsection{Response of DNA in Electric Driving Fields}

A summary of the various dielectric relaxations in DNA is given in figure 3.2. The lowfrequency response of DNA is due primarily to longitudinal polarization of the diffuse counterion sheath that surrounds the molecule depicted in figure 1.5. This occurs at frequencies in the range of 1 to $100 \mathrm{~Hz}$. Another relaxation occurs in the megahertz region due to movement of condensed counterions bound to individual phosphate groups. There are many other types of motion of the DNA molecule in response to mechanical or electrical driving fields. For example, propeller twist occurs when two adjacent bases in a pair twist in opposite directions. Another motion is the breather mode in which two bases oscillate in opposition as hydrogen bonds are compressed and expanded. The Lippincott-Schroeder and Lennard-Jones potentials are commonly used for modeling these motions. These modes resonate at wavelengths in the millimeter region; however, relaxation damping prevents direct observation. Other static or dynamic motions of the 


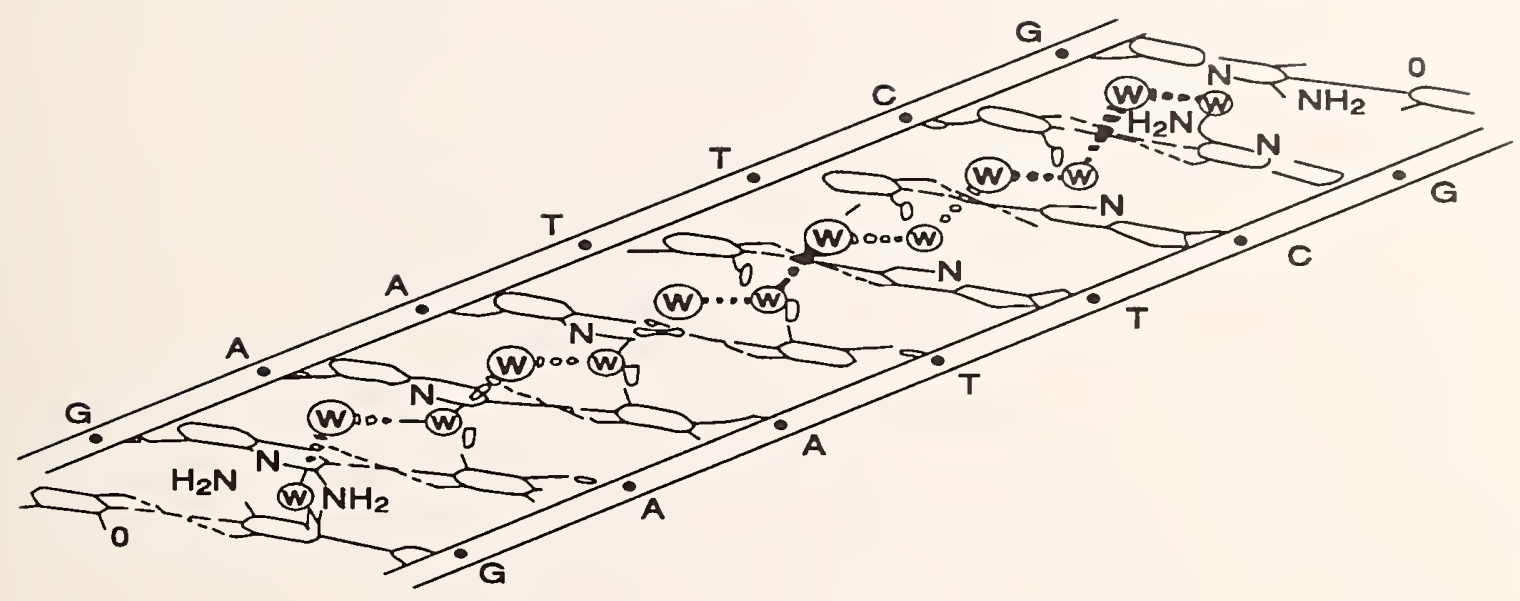

Figure 3.1. Water backbone in the minor groove of B-DNA. W denotes water molecule, dashed lines used for hydrogen bonds in base pairing. The water molecules are around the phosphate groups. 


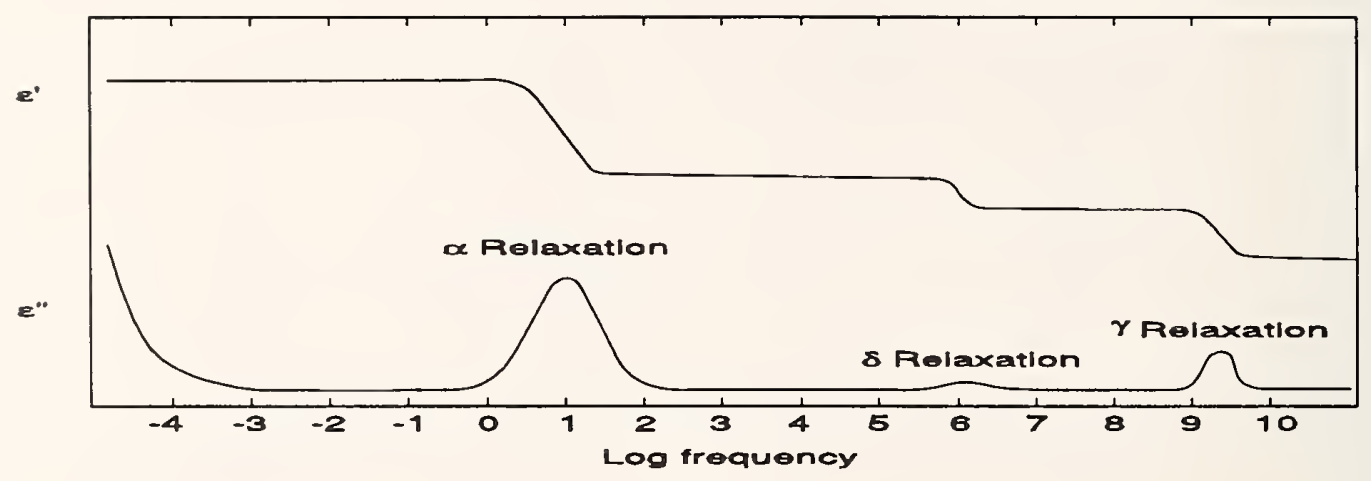

Figure 3.2. Dielectric relaxations of DNA.

base pairs of the DNA molecule are roll, twist, and slide.

A number of researchers have studied dielectric relaxation of both denatured and helical conformation DNA molecules in electrolyte solutions both as a function of frequency and applied field strength. Single-stranded DNA exhibited less dielectric relaxation than double-stranded DNA $[10,16,28]$. Takashima concluded that denatured DNA tended to coil and thereby decrease effective length and therefore dipole moment. Further, a high electric field strength affects DNA conductivity in two ways [28]. First, it promotes increased dissociation of the molecule and thereby increases conductivity. Second, it promotes an orientation field effect where alignment of polyions increases conductivity.

Single-stranded DNA, in its stretched state, possesses a dipole moment oriented more or less transverse to the axis. The phosphate group produces a permanent transverse dipole moment of about $20 \mathrm{D}$ per $0.34 \mathrm{~nm}$ base-pair section. Since the typical DNA molecule contains thousands of base pairs, the net dipole moment can be significant. However, as the molecule coils or the base pairs twist, the dipole moment decreases. If single strands of DNA were constrained to be rigid, relaxation would occur in the low megahertz frequencies. 


\subsection{Dynamics of Polarization Relaxation}

In order to study relaxation of DNA in solution we first consider the simplest model of a dipolar, rigid rod.

The torque on an electric moment $\vec{p}$ is

$$
\vec{N}=\vec{p} \times \vec{E}
$$

For cases where the dipole moment is perpendicular to the rod axis, rotations about the major axis can occur (see figure 3.3). The longitudinal rotation relaxation time is $[29]$

$$
\tau_{l}=\frac{K L}{2 k_{B} T}
$$

where $K$ is a constant, $k_{B}$ is Boltzmann's constant, and $L$ is the length of the molecule. The relaxation time varies as $L$. Major axis rotation could occur if the molecule had a transverse dipole moment, for example, in a single strand of DNA.

When the dipole moment is parallel to the major axis, that is when there is excess charge on the dipole ends, end-over-end rotation will occur as shown in figure 3.3. The end-over-end relaxation time for a rigid molecule of length $L$, viscosity $\eta_{0}, L / b \gg 1$, $\gamma=0.8$, and radius $b$ is $[22,23]$

$$
\tau_{e}=\frac{\pi \eta_{0} L^{3}}{6 k T[\ln (L / b)-\gamma]}
$$

This is the type of low-frequency relaxation that occurs with the induced dipole moment in the counterion sheath or a permanent dipole moment parallel to the longitudinal axis of the molecule. The relaxation time varies as $L^{3}$. Since length of the molecule and molecular mass are related, the responses for the two relaxations depend on molecular mass. Also, the model presented in this section assumed a rigid rod. In reality DNA is not rigid, so a statistical theory of relaxation needs to be developed. We will address these problems in a later section [29-31].

Takashima [16] and Sakamoto [10] have derived a more comprehensive theory for counterion relaxation and found that the relaxation time varies in proportion to the square of the length of the molecule $[31,32]$. Most experimental evidence indicates a $L^{2}$ dependence. This is in contrast to the rigicl-rod model where the relaxation time raries as $L^{3}$. 

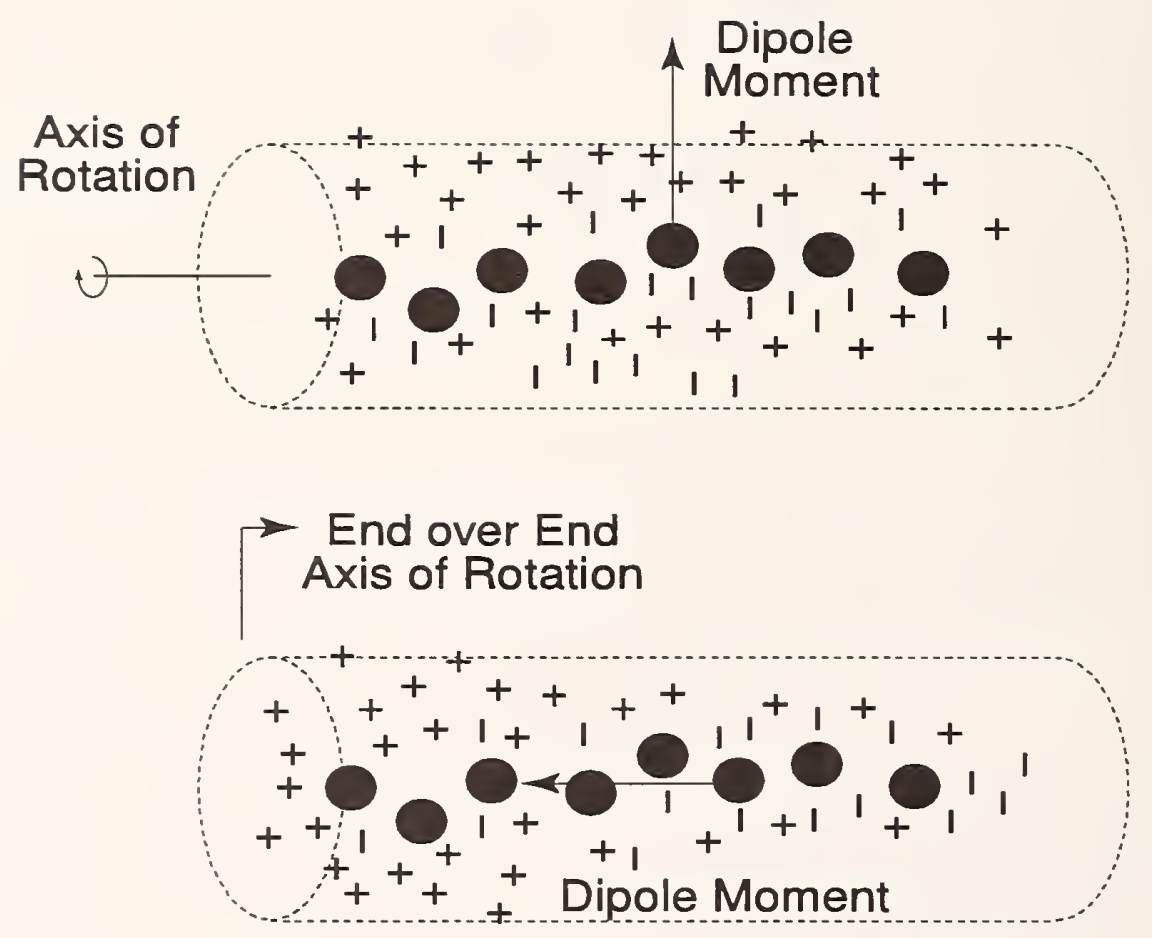

Figure 3.3. Torques on dipolar, rigid rod. 


\subsection{The Electric Field near the DNA Molecule}

There have been a number of studies on the electric field surrounding the DNA molecule $[33,34]$. Edwards et al. [33] studied a case where charge was considered to reside only on the sugar-phosphate backbone and bases. In these studies the molecule was represented by three concentric cylinders. The inner cylinder represented the molecule, the middle layer represented counterions, and the outer layer represented the solvent. The charges from the phosphate backbone were assumed to reside on the outer surface of the inner layer. In these studies the major and minor grooves were not modeled. The skin depth of the field, that is the distance over which the field attenuates to $1 / \mathrm{e}$ of its initial value, was on the order of $0.5 \mathrm{~nm}$. Beyond this distance, the field approached that of a line charge.

\section{Counterions and the Helix}

\subsection{Cations}

The permittivity depends on concentration and type of cations [32]. As the concentration of the solvent increases, more of the phosphate charge is neutralized and the dielectric increment decreases.

Many types of cations compounds have been used in DNA solvents, for example, $\mathrm{NaCl}, \mathrm{LiCl}, \mathrm{AgNO}_{2}, \mathrm{CuCl}_{2}, \mathrm{MnCl}_{2}, \mathrm{MgCl}_{2}$, arginines, protamine, dyes, lysine, histones, and divalent metals such as $\mathrm{Pb}, \mathrm{Cd}, \mathrm{Ni}, \mathrm{Zn}$, and $\mathrm{Hg}$. [10,35-37]. The simple inorganic monovalent cations bind to the DNA molecule near the phosphate backbone to form both a condensed and diffuse sheath. There is evidence that strong concentrations of divalent metal cations destabilize the DNA helix [38]. Sakamoto [36] found that the dielectric increment decreased for divalent cations. On the other hand, histones and protamines tightly bind in the major groove of the DNA molecule. They produce stability in the double helix by neutralizing some of the phosphate charge. Dyes can attach to DNA, neutralize charge, and thereby decrease dielectric increment. The cation concentration determines the dissociation of the DNA molecule. Mctague and Gibbs showed that the Manning theory predicts that the counterion dipole moment should increase as cation concentration increases $[39,40]$. In this respect the Manning theory contradicts experiment. The theory developed by Oosawa [19] yields more realistic predictions. In figures 4.1 through 4.3 plots of dielectric increment versus cation and 


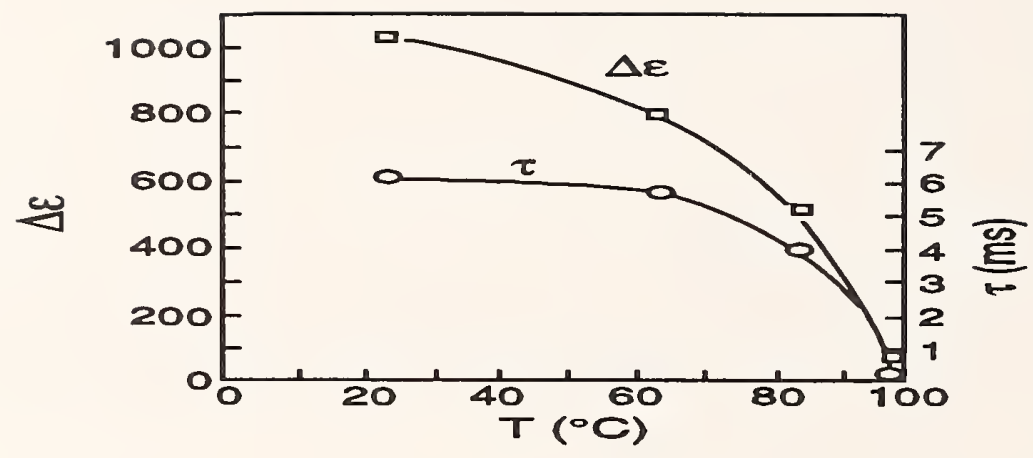

Figure 4.1. Effect of cations on relaxation time and dielectric increment [36].

DNA concentration are given $[10,32,35,36]$.

Both protamine and histones are proteins. Nucleohistones and protamine-DNA complexes occur in somatic-cell nuclei of mammals. Research on the dielectric properties of nucleohistones and DNA has been performed by Jungner [41]. Protamine is bound to DNA in the major groove by electrostatic interactions with phosphate charge [42]. Protamine has a total of 31 amino acid residues, with 67 percent arginine. Protamine stabilizes DNA by neutralizing the phosphate charges in the DNA molecule and thereby increasing the melting temperature $[43,44]$. The denaturing temperature of DNA in protamine increases because of the tight binding of protamine in the major groove. The use of protamine as a cation in DNA solutions allows a low-conducting fluid to stabilize the molecule. This is a useful feature in applications where conductivity is important.

Expression of gene components during embryonic development is controlled by proteins, such as protamine, which interact with segments of DNA. Proteins can locate a specific section of base pairs by recognition of specific bends and twists in the DNA molecule. Proteins can locate short base sequences from the billions of bases in the gene by interacting with DNA by attaching to DNA helices in the major and minor 


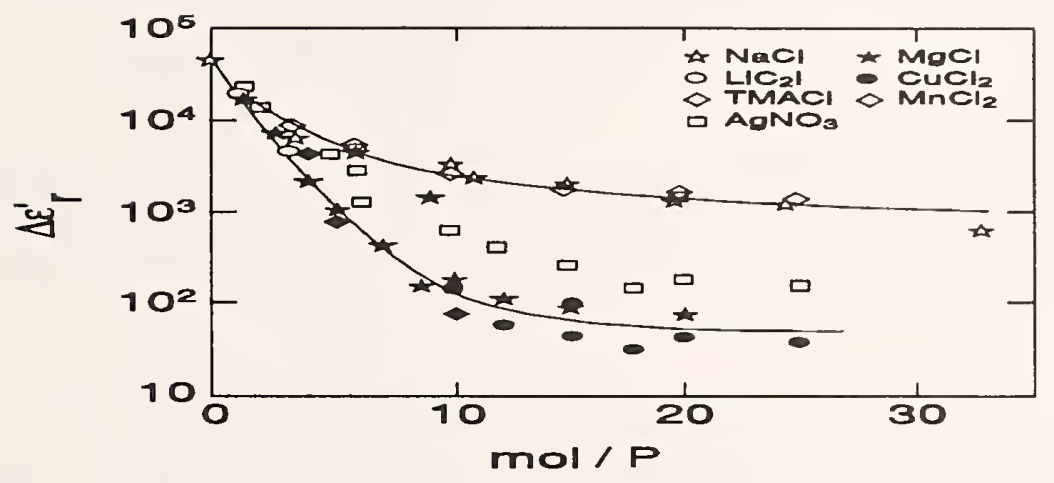

Figure 4.2. Dielectric increment versus mol/P where $\mathrm{P}$ is number of phosphate residues [36].

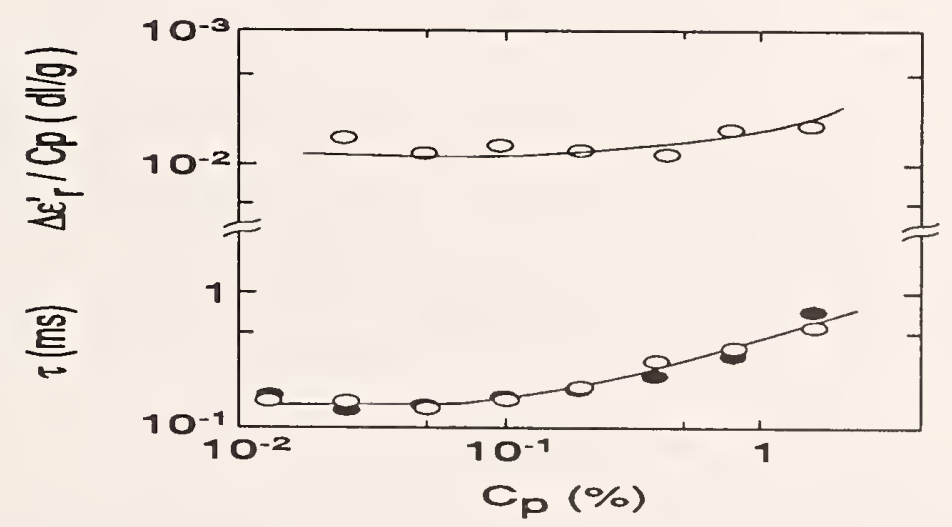

Figure 4.3. Dielectric increment and relaxation time is concentration of DNA $C_{p} .36$ 
grooves. Bound water is very important in the DNA-protein interaction since it is used in hydrogen bonding.

An empirical fit of the melting temperature $T_{m}$ of protamine-DNA solutions as a function of salt concentration has been derived [37, 45]

$$
T_{m}=T_{m}^{0}+C_{p} \log N a^{+}
$$

Here $\mathrm{Na}^{+}$is sodium molar concentration, $C_{p}$ and $\mathrm{T}_{m}^{0} \approx 92{ }^{\circ} \mathrm{C}$ are constants. We can compare this melting curve to that of DNA in saline solution. The helix melting temperature in saline solution in degrees Celsius is related to the concentration of salts by $[3,46]$

$$
T_{m}=100.3+14.8 \ln C_{p},
$$

where $C_{p}$ is concentration in moles per liter. This expression has been obtained by both empirical means and derived from helix models. DNA in saline solution melts at a lower temperature than in protamine solutions.

Bonincontro et al. $[37,45]$ investigated the effects of protamine sulphate (clupeine) and arginine on dielectric relaxation for herring sperm both as a function of temperature $\left(5^{\circ} \mathrm{C}\right.$ to $\left.40^{\circ} \mathrm{C}\right)$ and over a frequency range of $1 \mathrm{MHz}$ to $10 \mathrm{GHz}$. They fitted the relaxation data to a Cole-Cole model and then estimated the relaxation time $\tau$. Bonincontro [45] found that clupeine in saline solution showed relaxation behavior. Further, they mixed clupeine with DNA, the relaxation properties of DNA decreased. This indicates that clupeine decreases the induced polarization by neutralizing phosphate charges. They also concluded that the binding of protamine to DNA reduces the number of water molecules in the DNA complex [45]. Since protamine is a major-groove binder (see figure 4.4) it tends to exclude bound water from the groove [45]. Bonincontro also observed that relaxation was diminished when protamine was added to DNA solution. They concluded this is due to the compaction of the DNA caused by the protamine and consequential charge neutralization. The following model was used for the permittivity

$$
\epsilon^{*}=\epsilon_{\infty}+\frac{\Delta \epsilon_{1}}{\left(1+\left(j \omega \tau_{1}\right)^{1-\alpha}\right)}+\frac{\Delta \epsilon_{w}}{\left(1+j \omega \tau_{w}\right)},
$$

where $\Delta \epsilon_{1}$ and $\Delta \epsilon_{w}$ indicate dielectric increments for DNA and water respectively, $\tau_{1}, \tau_{w}$ are relaxation times, $\epsilon_{\infty}$ is the optical limit permittivity, and $\alpha$ is a Cole-Cole parameter. They obtained the activation enthalpy of the clupeine interaction from a plot of 


$$
\frac{1}{\tau T}=\frac{K}{\tau} \exp (\Delta S / R) \exp (-\Delta H / R T)
$$

where $\Delta H$ is the enthalpy of activation energy, $K$ is a constant, and $\Delta S$ is the molar entropy of activation energy. Equation (4.4) can be used to estimate the activation enthalpy since the slope of the plot of $1 / \ln (\tau T)$ versus. $1 / T$ yields $-\Delta H / R$.

\subsection{Helix-Coil Transition in DNA}

DNA can be either in the helical state or in the single-strand random-coil state. In the random-coil state, the hydrogen bonds that hold the helix together are broken. The state that prevails depends on cation concentration and temperature of the solvent $[3,46-48]$. The stability of the helix is determined from intermolecular interactions such as hydrogen bonds between base pairs, stacking of base pairs, interactions between bound and free-water molecules, water in the hydrate regions of DNA, and hydrophobic forces. The helix stability depends on ion concentration since the counterions neutralize charge and thereby decrease repulsion between phosphate groups. Stability also depends on temperature since as kinetic energy increases, the probability that hydrogen bonds will break increases according to an Arrhenius-type law. The presence of bound water in the molecule also produces stabilization.

The hydrogen base-pair bonds are not the primary force that holds the DNA strands together. It is thought that the helix structure is stabilized by water molecules [13]. Thermal denaturation destroys the ordered hydrate structure that holds the helix together. Electrostatic interactions oppose base stacking, whereas nonpolar interactions due to hydrophobic effects and van der Waals forces drive base stacking [49].

The helix-coil transition occurs at certain threshold values of $\mathrm{pH}$, salt concentration, or temperature. Increasing the $\mathrm{pH}$ above 7 will tend to denature polynucleotides. Denaturation readily occurs at pH's in excess of 12 or less than 2 due to base hydrogenbond ionization. The reason for this is that at higher $\mathrm{pH}$ the bases lose protons. A double helix cannot be formed by deprotonated bases since the protons lost are vital in the base-pairing reactions. The transition temperature depends strongly on saline concentration and $\mathrm{pH}[50]$. Stronger concentrations yield a higher dissociation temperature. This is due to the screening of the phosphate charges by the saline solution [46]. The helix-coil transition depends strongly on the free energy of the molecule together 


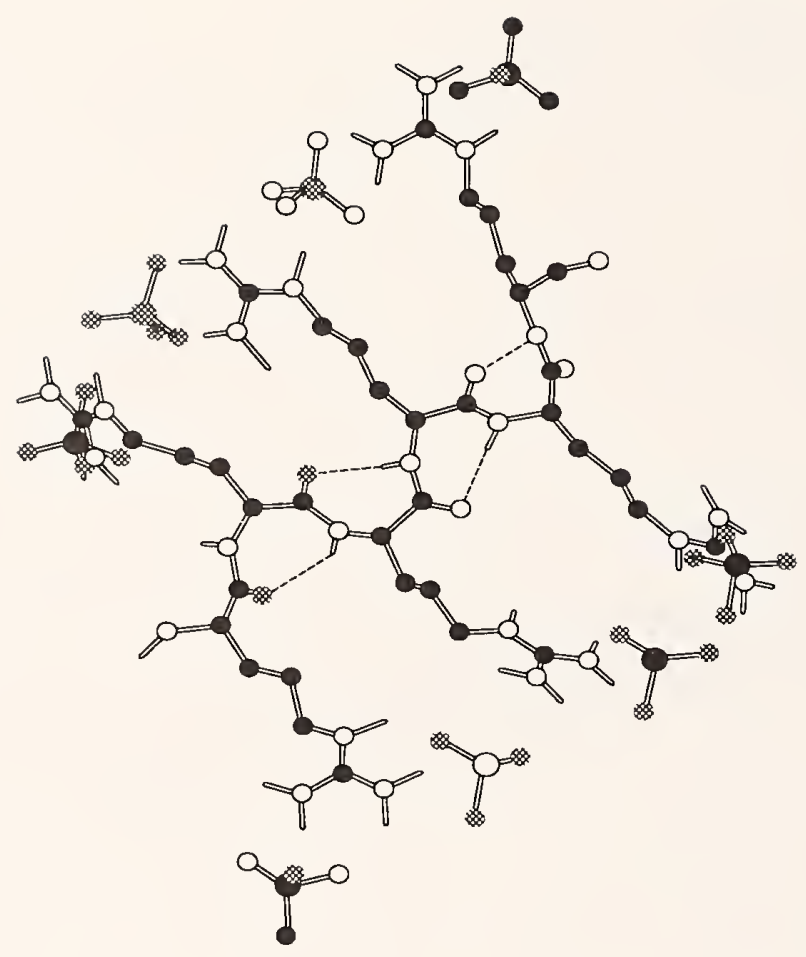

Figure 4.4. Six arginine residues bound to DNA; only the phosphate groups of the DNA molecule are shown. 


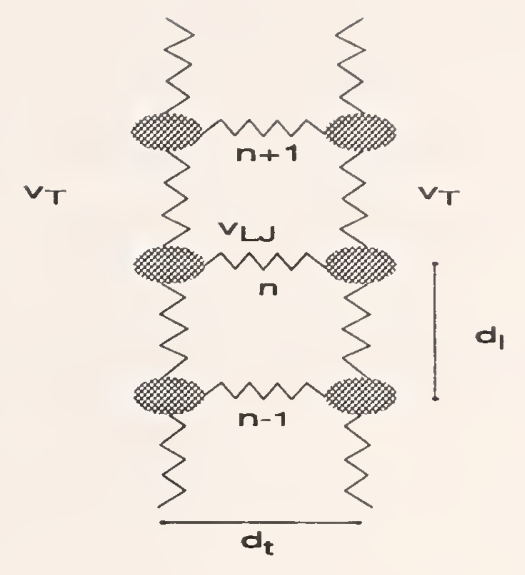

Figure 4.5. Denaturing process modeled by Toda (T) and Lennard-Jones (LJ) potentials.

with solvent. The molecule in each of its states has different free energy. In thermal denaturation counterions are released from the molecule as the strands separate. This increases the conductivity of the solvent.

It is well-known that Type-B is more stable than Type-Z DNA. This is due to the fact that the phosphate charge in Type-B DNA is positioned so that it gets more counterion screening than in Type-Z DNA [6].

A number of approaches have been used to model the denaturing of DNA. lakushevich [51] presented a simplified model of DNA where the bonds were modeled by springs using the sine-Gordon equation. Muto and others [52-55] modeled the melting process by considering the helix backbone to be composed of anharmonic Toda potentials between nucleotides and Lennard-Jones potentials for the hydrogen crosslinks as shown in figure 4.5. In this model the bonds were modeled by potentials and the coupled-force problem was solved numerically. Thermalization was included in the model by introducing white noise. The analytical solutions to the resultant differential equations are thermal solitons. This approach is primitive in that the counterion atmosplere is not adequately modeled.

Galindo [46] modeled the transition by considering the helix in two states. The first 
state was mincoiled double-stranded DNA consisting of two parallel chains of charges; in the other state the helix was coiled. The free energy for each of the states was calculated. The minimum of free energy $f$ was assumed to occur at equilibrium. It was useful to define the free energy difference between two conformations $\mathrm{X}$ and $\mathrm{Y}$ by

$$
\Delta f(X, Y)=\sum_{i}\left[f_{i}(X)-f_{i}(Y)\right] .
$$

The index $i$ denotes the different free energies of the different conformations. The most probable conformation was found by minimizing $\Delta f$.

\section{Modeling the Potential around the DNA Molecule}

\subsection{Phenomenological Models of DNA Dynamics}

Two dissimilar materials can have different electronic affinities. When they are placed in contact frequently a potential gradient between materials develops which allows current to flow. As a result an electrical double layer forms at a material interface. This interface could be between liquid and metal electrode or the layer between DNA and liquid. Smaller electron affinity will acquire greater positive charge. The potential difference will attract ions of opposite charge to the surface and repel like charges. The potential decreases roughly exponentially from the surface as $\psi(x)=\psi_{0} \exp \left(-x / \lambda_{D}\right)$, where $\lambda_{D}$ is the Debye screening length or skin depth. The region near the electrode consists of the Stern layer and a diffuse region which is beyond the Stern layer. A detailed analysis of the potential requires the solution to the Poisson-Boltzmann equation.

In the past, Manning's model, and various extensions of it, have been used to explain the counterion interaction and dielectric relaxation of DNA $[56,57]$. More recently the Poisson-Boltzmann equation has been used [58]. In the Manning model two distinct dispersions are assumed to occur in dissolved DNA. One dispersion is at low frequencies due to end-over-end relaxation of the sheath (see figure 3.3). The lower frequency dispersion occurs around 1 to $100 \mathrm{~Hz}$ and is due to counterion fluctuations along the sheath of the polymer. This relaxation depends on the polymer length or molecular mass. A dispersion at higher frequencies due to relaxation of subunits of counterions in the sheath has also been observed (see figure 3.2). This relaxation does not depend on molecular mass. Mandel developed a model based on Manning's work where the biopolymer was considered to be a long string of hinged, rigid rods [56,57]. Oosawa 


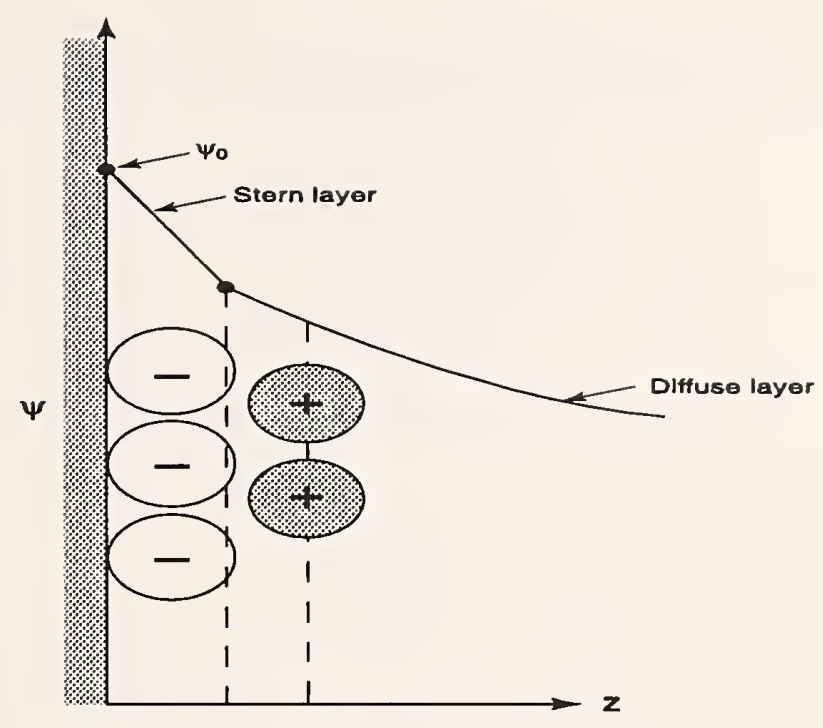

Figure 5.1. Electrode with electrolyte

incorporated a more complicated model by solving the diffusion equation, as in Mandel's model, but included surface diffusion of condensed counterions and two rotational modes of DNA $[19,59]$.

\subsection{Poisson-Boltzmann Equation}

The Poisson-Boltzmann equation is commonly used for modeling the counterion atmosphere around a charged object $[3,46,60,61]$. In this section we review the timeindependent theory for counterions in an applied potential. Dukhin and Shilov [62] present a very detailed analysis of the counterion atmosphere and dielectric relaxation around electrodes.

We consider counterions near an electrode (see figure (5.1)). We can develop a differential equation for the potential $\psi(\vec{r})$ if we substitute

$$
\vec{E}=-\nabla \psi
$$

into

$$
\nabla \cdot \vec{D}=\rho
$$

to form

$$
\nabla^{2} \psi+\frac{\rho}{\epsilon}=0
$$


where $\rho$ is the charge density of the counterions, $\epsilon$ is the permittivity of the solvent and is assumed constant in space. The counterion charge density is written using the Boltzmann distribution $n^{ \pm}(\vec{r})=n_{0} \exp \left(\mp z_{c} q \psi(\vec{r}) / k_{B} T\right)$, where $r$ is the coordinate, $z_{c}$ is the valency per ion, $q$ is the charge per ion, $n_{0}$ is the ion density at infinity, $k_{B}$ is Boltzmann's constant, and $T$ is the temperature. The net charge density for a single species of ion in one dimension is the sum of negatively and positively charged ions

$$
\begin{gathered}
\rho(z)=z_{c} q\left(n^{+}(z)-n^{-}(z)\right)=-2 n_{0} z_{c} q \sinh \left(\frac{z_{c} q \psi(z)}{k_{B} T}\right), \\
k^{2}=\frac{2 n_{0} z_{c}^{2} q^{2}}{\epsilon k_{B} T} .
\end{gathered}
$$

The Poisson-Boltzmann eq. (5.3) in one-dimensional normalized rectangular coordinates can be written

$$
\frac{d^{2} y}{d z^{2}}=k^{2} \sinh (y)
$$

where the normalized potential is

$$
y=\frac{z_{c} q \psi}{k_{B} T}
$$

and $\mathrm{k}$ is the reciprocal of the Debye screening length.

The physical significance of the Debye screening length $k$ is to produce damping. As an example, a liquid with an ion density $n_{0}=6 \times 10^{24} \mathrm{~m}^{-3}$ and $\epsilon_{r 0}^{\prime}=78$ yields $k \approx 2 \times 10^{8} \mathrm{~m}^{-1}$. This value of $1 / k$ corresponds to a skin depth of $5 \mathrm{~nm}$.

The boundary conditions for eq. (5.6) are

$$
y(z=0) \equiv y_{0}=\frac{z_{c} q \psi_{0}}{k_{B} T}
$$

where $\psi_{0}$ is the potential on the boundary. At $\infty$

$$
\begin{gathered}
y(z \rightarrow \infty) \rightarrow 0 \\
\frac{d y}{d z}(z \rightarrow \infty) \rightarrow 0 .
\end{gathered}
$$

The first integral is $[63]$

$$
\frac{d y}{d z}=-2 k \sinh (y / 2)
$$

and the second is

$$
y(z)=4 \tanh ^{-1}\left[\tanh \left(y_{0} / 4\right) e^{(-k z)}\right]
$$


or

$$
y(z)=\log \left[\frac{e^{\left(y_{0} / 2\right)}+1+\left(e^{\left(y_{0} / 2\right)}-1\right) e^{-k z}}{e^{\left(y_{0} / 2\right)}+1-\left(e^{\left(y_{0} / 2\right)}-1\right) e^{-k z}}\right],
$$

where

$$
y_{0}=\frac{z_{c} q \psi_{0}}{k_{B} T} .
$$

The surface-charge density and differential capacitance are

$$
\sigma=2 \sqrt{2 k_{B} T \epsilon n_{0}} \sinh \left(\frac{z_{c} q \psi_{0}}{2 k_{B} T}\right)
$$

and

$$
C_{d}=\sqrt{2 \frac{z_{c}^{2} q^{2} \epsilon n_{0}}{k_{B} T}} \cosh \left(\frac{z_{c} q \psi_{0}}{2 k_{B} T}\right) .
$$

Equations (5.15) and (5.16) do not yield physical results since they both increase exponentially with $\psi_{0}$. Corrections are commonly made to the Poisson-Boltzmann equation in the analysis by including an extra layer, commonly called the Stern layer, that varies linearly with potential.

\subsection{Poisson-Boltzmann Applied to Modeling the DNA Helix}

It is possible to use the Poisson-Boltzmann equation to study the counterion atmosphere around the DNA helix by assuming a charge distribution or potential on the helix surface. A number of models have been developed to study the helix and helix-coil transition. For example, the model by Galindos [46] assumes a counterion atmosphere and a helix of infinite length with constant charge density distributed evenly on the surface. In this study they assumed two cylindrical layers. The first layer contained the condensed counterions and in the second layer, the diffuse counterions. This is an approximation since most of the charge actually occurs in the phosphate groups in the backbone. Each phosphate-sugar group was assumed to have one electron of extra charge. The boundary conditions on the surface of the DNA at $r=a$ are Gauss' law

$$
\frac{d \psi}{d r}(a)=\frac{2 q_{l}}{a}
$$

and the vanishing of the field at $r \rightarrow \infty$

$$
\psi(\infty)=0
$$


$q_{l}=\mathcal{L}_{b} / b$, where $b$ is the length of the cylinder over which the charge is distributed and $\mathcal{L}_{b}$ is the Bjerum length. The solution in this case for the potential around the helix is

$$
\psi(r)=\frac{k_{B} T}{q} \ln \frac{k^{2} r^{2}}{2\left(\epsilon_{s} / \epsilon_{b} \beta_{0}^{2}\right)} \sinh ^{2} \beta_{0} \ln (A r),
$$

where $\epsilon_{b}$ and $\epsilon_{s}$ are the dielectric constants of the bulk solvent and solute and $A$ and $\beta_{0}$ are constants which are determined by the boundary conditions. It is important to realize that the dielectric constant near the surface is different than it is far away from the surface. The state of conformation is determined by minimizing the free energy as described in Section 4.2.

\subsection{Numerical Models for DNA Dynamics}

A number of extensive numerical models have been developed that solve equations for the electric field, counterion dynamics, and DNA charge sources [7,64,65]. Models such as these are useful in predicting behavior of the DNA molecule. Saxena [66] has developed a comprehensive numerical model for dissolved DNA dynamics. In his model equations of motion for DNA and the counterion sheath are solved numerically in the frequency domain for the individual atoms and the counterion sheath. The model by Saxena and Van Zandt contains a comprehensive model of site and sheath-bound counterions [9]. This study was limited to the microwave and millimeter-wave frequencies and therefore low-frequency dispersion was not studied. Examples of equations of motion are $[9]$

$$
\begin{aligned}
& \underbrace{\frac{d^{2} q_{i}^{\alpha}}{d t^{2}}=\sum_{j, \beta} D_{i j}^{\alpha \beta} q_{j}^{\beta}+e_{i} E_{\alpha}+\Gamma_{i}\left[\frac{d s}{d t}-\eta_{i} \frac{d q_{i}^{\alpha}}{d t}\right] \delta_{i P} \delta_{\alpha z}}_{\text {atoms }}, \\
& \underbrace{\frac{d^{2} s}{d t^{2}}=-q^{2} v_{w}^{2} s-\lambda E_{z}+\sum_{i} \Gamma_{i}\left[q_{i}^{z}-s / \eta_{i}\right] \delta_{i P}+\gamma \frac{d s}{d t}}_{\text {sheath }},
\end{aligned}
$$

where $q_{i}^{\alpha}$ are the displacements of atom $i$ and component $\alpha, s$ represents the displacement of the sheath, $D_{i j}$ is a force-constant matrix containing van der Waals, Coulombic, and elastic forces, $v$ is velocity, $\lambda$ and $\Gamma$ are constants, and $\eta$ is viscosity. The last term on the right of eq. (5.20) represents the polymer-solvent dissipation force. In these 
equations only longitudinal motion of the sheath is considered. Specific forms for the electric field in the various cylindrical regions are assumed.

\section{Phenomenological Models of Dipole Moment, Relaxation Time, and Polarizability}

\subsection{Persistence Length and Radius of Gyration}

Double-stranded DNA is in the form of a meandering chain. Since the DNA molecule is not entirely rigid, the induced dipole moment of the counterion sheath depends on the persistence length rather than the actual length. The persistence length of a chain of atoms is the average projection of the end-to-end distance vector on the first bond in the chain. It is a measure of how the final direction of a chain deviates from the initial link of the chain. The radius of gyration gives an effective radius of the molecule. It is a measure of the mean distance from the center of gravity. The radius of gyration depends on the structural integrity of the molecule, that is, the degree of dissociation of the molecule. Sakamoto derived the following formula for dipole moment of DNA as a function of the mean-squared radius of gyration $\left\langle S^{2}\right\rangle$, the number of phosphate residues $n$ and the degree of dissociation of the molecule $\alpha_{B}[32]$.

$$
<\mu^{2}>=n \alpha_{B}\left(1-\alpha_{B}\right) q^{2}<S^{2}>\text {. }
$$

Sakamoto also studied protein (histone) attachment to DNA and derived an empirical formula for the dipole moment in terms of radius of gyration [36]

$$
<\mu^{2}>=m\left(1-\frac{m}{n}\right) q^{2}<S^{2}>
$$

Here $m$ is the number of positively charged residues of proteins attached to the DNA. In eqs. (6.1) and (6.2), $<S^{2}>$ was calculated using the Kratky-Porod equation in terms of the persistence length $l_{p}$ and contour length $L$ for a worm-like chain [67]

$$
<S^{2}>=\frac{l_{p} L}{3}-l_{p}^{2}+\frac{2 l_{p}^{3}}{L}-\frac{2 l_{p}^{4}}{L^{2}}+\frac{2 l_{p}^{4}}{L^{2}}\left[1-\exp \left(-L / l_{p}\right)\right] .
$$

\subsection{Polarization}

Consider a rodlike polyion surrounded by a counterion atmosphere [68]. Let $\delta$ be the instantaneous displacement between counterion and polyion charges. Then the net 
dipole moment is $\mu=\sum_{i} q_{i} \delta_{i}$. The polarizability can be calculated from the mean dipole moment

$$
<\mu>=\frac{\int \mu \exp \left(-f(\mu) / k_{B} T+\vec{\mu} \cdot \vec{E} / k_{B} T\right) d \mu}{\int \exp \left(-f(\mu) / k_{B} T+\vec{\mu} \cdot \vec{E} / k_{B} T\right) d \mu},
$$

where $f$ is the free energy and $\vec{E}$ is the electric field. The polarizability is defined in eq. (3.1). In the absence of a field, the average dipole moment $\langle\mu\rangle=n q\langle\delta\rangle=0$ where $n$ is the number of charge sites. However, the fluctuations in the dipole moment are nonzero and are the origin of polarizability.

There are a number of models for DNA polarizability. The model developed by Mandel [56] overestimates the counterion induced polarizability since it neglects counterion repulsion. The model of Manning [69] predicts an increase in polarizability with increasing solvent concentration, in contradiction to experiment [70]. Polarizability decreases with increasing solvent strength [71]; probably this is due to phosphate charge neutralization by the increased concentration of counterions. Polarization also decreases with increasing temperature.

Oosawa developed a comprehensive time-dependent theory of polarization for bound counterions using a diffusion equation $[19,59]$. This model predicts the relaxation frequency for the sheath. The induced dipole moment forms due to fluctuations in the sheath density. In this theory, $\delta=\sum_{i} x_{i} / N$ is the displacement of the center of gravity of $N$ counterions. The collective motion of the counterion atmosphere is given by the simple diffusion equation

$$
N \zeta \frac{\partial \delta}{\partial t}+\frac{k_{B} T}{<\delta^{2}>} \delta=0
$$

where $\zeta$ is a frictional constant. Equation (6.5) has solution

$$
\delta(t)=A \exp (-t / \tau)
$$

where the relaxation time is $\tau=N \zeta<\delta^{2}>/ k_{B} T$. In a more comprehensive theory, Oosawa included a full-mode spectrum. This theory predicts that the relaxation time is proportional to the square of the polyelectrolyte length and inversely proportional to temperature. These predictions are consistent with experimental results.

In another model of polarizability, Fixman assumed DNA to be an impenetrable cylinder [72] and solved the Poisson-Boltzmann equation for the counterion distribution in an external field. His model produced an expression for the induced dipole moment 
by integrating the charge density over the length of the molecule. The Fixman model underestimates the polarizability, but correctly predicts the solvent dependence of the polarizability. The advantage of the Fixman model is its simplicity. The Fixman model yields the following expression for polarizability

$$
\alpha=\frac{\epsilon L K_{f}}{\nu^{2}}\left(\frac{z_{1}}{z_{1}-z_{2}}\right)\left[1-\frac{2 \tanh (\nu L / 2)}{\nu L}\right]
$$

where $L$ is molecule length, $\nu^{2}=\pi N_{A} c_{1} K_{f} / 250 \sigma, K_{f}=(2 \ln (2 L / a)-14 / 3)^{-1}, \epsilon$ is permittivity, $z_{1}, z_{2}$ are charges on counterions and coions, $N_{A}$ is Avogadro's number, $c_{1}$ is the concentration of counterions, $a \approx 1.2 \mathrm{~nm}$ is radius of the molecule, $\sigma=(1-1 / \zeta) / b^{\prime}$, $\zeta=q^{2} / b^{\prime} \epsilon k_{B} T, b^{\prime}=b /(1-\rho)$, and $b$ is length of base pair.

In a final example of polarization models. Hogan proposed a model where the dipole moment is formed due to anisotropic counterion flow along the axis of the molecule [17].

\subsection{Simple Model of Dipole Moment}

The dipole moment of polymer molecules is usually modeled by a set of charges that are connected by flexible links [32]. In this section, we review a model for the dipole moment of a string of rigid rods [32]. The net charge (see figure 6.1) at positions $\vec{r}_{i}$ is $q \delta n_{i}=q\left(n_{i}-<n>\right)$, where $q<n>$ is the mean value of charge distribution. The net dipole moment is

$$
\vec{\mu}=\sum_{i} q\left(\vec{r}_{i}-\vec{r}_{G}\right) \delta n_{i}
$$

where the center of mass for $N$ links is

$$
\vec{r}_{G}=\frac{1}{N} \sum_{i} \vec{r}_{i} .
$$

The mean-squared moment is

$$
<\mu^{2}>=<q^{2} \sum_{i} \sum_{j}\left(\vec{r}_{i}-\vec{r}_{G}\right)\left(\vec{r}_{j}-\vec{r}_{G}\right) \delta n_{i} \delta n_{j}>
$$

This is approximately

$$
<\mu^{2}>=q^{2} \sum_{i} \sum_{j}\left(\vec{r}_{i}-\vec{r}_{G}\right)\left(\vec{r}_{j}-\vec{r}_{G}\right)<\delta n_{i} \delta n_{j}>.
$$




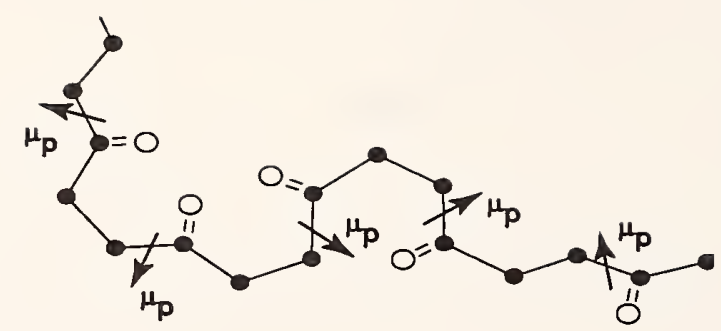

Figure 6.1. Polymer chain of dipoles of moment $\mu_{p}$. 
If we apply this model to the double-stranded DNA helix and assume there is a probability $\alpha_{B}$ of an ion binding at a site, then the correlation function for the charge distribution can be approximated by

$$
<\delta n_{i} \delta n_{j}>=\delta_{i j} \alpha_{B}\left(1-\alpha_{B}\right)
$$

We then have

$$
\sqrt{<\mu^{2}>}=q \sqrt{N \alpha_{B}\left(1-\alpha_{B}\right)<L^{2}>}
$$

where

$$
<L^{2}>=\frac{1}{N} \sum_{i=1}^{N}<\left(\vec{r}_{i}-\vec{r}_{G}\right)^{2}>.
$$

This model correctly predicts the dependence of the dipole moment on the length of the polymer, but not the temperature dependence. In the next section we will generalize this model to get correct temperature dependence.

\subsection{Dipole Moment, Permittivity, and Relaxation Time}

Minakata developed a comprehensive, phenomenological model for discrete counterion binding on rod-like polymers [73]. It includes three different types of counterion binding sites. This model is important since it correctly predicts a number of the important measurable quantities in the relaxation process. The mean-squared dipole moment is defined as

$$
<\mu^{2}>=q^{2} \sum_{i j} r_{i} r_{j}<\left(n_{i}-<n>\right)\left(n_{j}-<n>\right)>=q^{2} \sum_{i}\left(\Delta_{i}^{2} n r_{i}^{2}+2 e^{2} \sum_{i j}\left(\Delta_{i j} n\right) r_{j} r_{i+j}\right)
$$

where $n$ is the number of bound ions at a specific site, $\Delta_{i}^{2} n=<n_{i}^{2}>-<n>^{2}$, and $\Delta_{i m} n=<n_{i} n_{i+m}>-<n^{2}>$. This model yields

$$
\Delta \epsilon=\frac{\pi N B n q^{2} L^{2}}{9 k_{B} T}\left[\Delta n^{2}+2 \sum_{i} \Delta_{i} n\right] .
$$

Here $B$ is the ratio of the internal to external fields, $L$ is length of the molecule, and $N$ is number of polyions per unit volume. The important result of this model is that it correctly predicts the dielectric increment to be proportional to $L^{2}$ and temperature dependence to be of the form $1 / T$. 


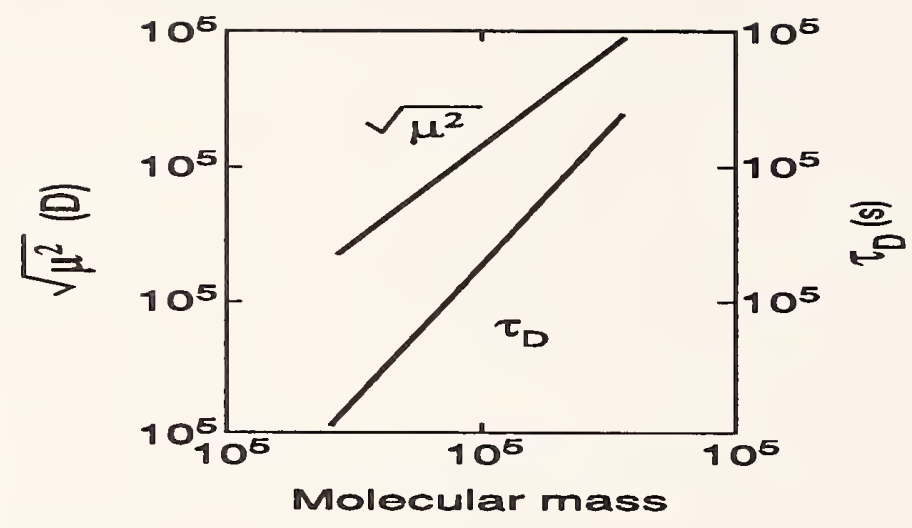

Figure 6.2. Relaxation time and dipole moment versus molecular mass.

Another model developed by Sakamoto [32], relates dipole moment of the counterion sheath to dielectric increment from counterion fluctuation theory and agrees well with experiment [32]

$$
\Delta \epsilon=\frac{4 \pi N<\mu^{2}>}{3 k_{B} T} .
$$

Sakamoto defined the dielectric relaxation time in terms of the maximum frequency $f_{m}$ in the loss spectrum by $\tau_{D}=1 / 2 \pi f_{m}$. For double-stranded calf thymus DNA, $\tau_{D}$ was proportional to the square of the molecular mass of water $M_{w}$ and the mean dipole moment was proportional to $M_{w}^{3 / 2}$ (see figure 6.2). He also found that for coiled DNA, $\tau_{D} \approx 2 \tau_{Z}$, where $\tau_{Z}=0.42 M_{w} \eta_{s} \eta_{r e d} / R T$ is the Zimm viscoelastic relaxation time (see figure 6.3). Here $\eta_{s}$ is solvent viscosity and $\eta_{\text {red }}$ is the reduced viscosity.

\subsection{Statistical-Mechanical Model of Polarization for Rod-Like DNA}

In this section we consider a more rigorous model of coupled DNA and counterion dynamics [68]. We solve a generalized diffusion equation or Fokker-Planck equation for the counterion sheath dynamics in an applied electric field and we develop new moment equations from the model. The model yields relaxation times and time-evolution equations. 


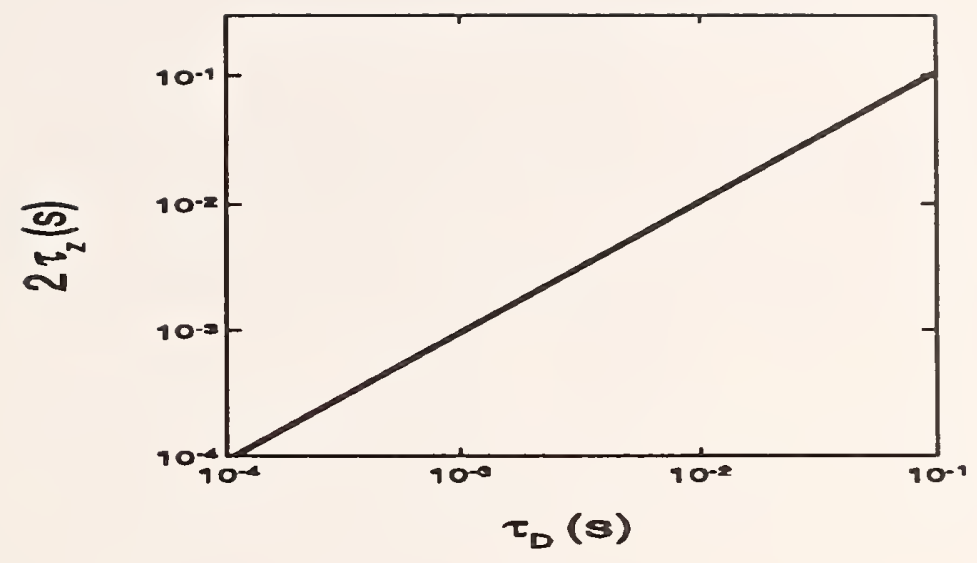

Figure 6.3. Dielectric relaxation time $\left(\tau_{D}\right)$ versus Zimm relaxation time $\tau_{Z}$.

The instantaneous dipole moment is $\mu=n q \delta$, where $\delta$ is the displacement along the major axis of the molecule from the center of charge distribution and $q$ is electronic charge. In addition there may be a permanent dipole moment $\mu_{p}$. The polarizability can be expressed in terms of the fluctuations $\alpha=\left\langle\mu^{2}\right\rangle / k_{B} T=n^{2} q^{2}<\delta^{2}>/ k_{B} T$. The potential energy of the counterion sheath in the absence of an electric field is

$$
V(\delta)=n^{2} q^{2} \delta^{2} / 2 \alpha
$$

This potential-energy function is quadratic in displacement analogous to the harmonic oscillator potential. Using this potential, the Fokker-Planck equation, which gives the probability that a certain counterion distribution will be realized, can be written as

$$
\frac{\partial p(\delta, t)}{\partial t}=D \frac{\partial}{\partial \delta}\left[\frac{\partial p(\delta, t)}{\partial \delta}+\beta\left(\frac{\partial V(\delta)}{\partial \delta} p(\delta, t)\right)\right]
$$

Here $\beta=1 / k_{B} T$ and $D$ is a diffusion constant. The steady-state solution is Gaussian

$$
p_{e q}(\delta)=\frac{\exp (-\beta V)}{\int_{-\infty}^{\infty} d \delta \int_{0}^{\pi} d \theta \sin \theta \exp (-\beta V)} .
$$

If we perform the integrations, we obtain the Gaussian distribution

$$
p_{e q}(\delta)=\sqrt{\frac{\beta \pi^{2} q^{2}}{2 \pi \alpha}} \exp \left(-\beta n^{2} q^{2} \delta^{2} / 2 \alpha\right) .
$$


Since the solution is Gaussian the average dipole moment will be 0 . The mean-squared dipole moment, $\left\langle\mu^{2}\right\rangle=k_{B} T \alpha$, however, is nonzero. The time-dependent solution of eq. (6.19) is

$$
p(\delta, t)=\sqrt{\frac{1}{2 \pi \tau D(1-\exp (-2 t / \tau)}} \exp \left(-\frac{(\delta-\delta(0) \exp (-t / \tau))^{2}}{2 D \tau(1-\exp (-2 t / \tau))}\right),
$$

where $\tau=\alpha / D \beta n^{2} q^{2}$. If we multiply eq. (6.19) by $\delta$ and then integrate from $[-\infty, \infty]$ we obtain

$$
\frac{d<\delta>}{d t}=-\frac{<\delta>}{\tau}
$$

with solution

$$
<\delta>(t)=\delta(0) \exp (-t / \tau)
$$

In the limit of large time, the mean dipole moment tends to 0 . In the absence of an electric field this behavior would be expected. The mean-squared dipole moment is

$$
<\delta^{2}>(t)=\frac{\alpha k_{B} T}{n^{2} q^{2}}+\left(\delta^{2}(0)-\frac{\alpha k_{B} T}{n^{2} q^{2}}\right) \exp (-t / \tau) .
$$

We see that this approximation has a single relaxation time $\tau$. In the limit of large time $<\delta^{2}>\rightarrow \alpha k_{B} T / n^{2} q^{2}$.

In the case where there is an applied electric field on the counterions in an anisotropic polyion atmosphere, the Fokker-Planck equation for rotational diffusion is

$$
\begin{aligned}
& \frac{\partial p(\delta, \theta, t)}{\partial t}=\left[\frac { \Theta } { \operatorname { s i n } \theta } \frac { \partial } { \partial \theta } \operatorname { s i n } \theta \left[\frac{\partial p(\delta, \theta, t)}{\partial \theta}+\beta\left(\frac{\partial V(\delta, \theta)}{\partial \theta} p(\delta, \theta, t)\right]\right.\right. \\
& \quad+D \frac{\partial}{\partial \delta}\left(\frac{\partial}{\partial \delta}+\beta\left(\frac{\partial V(\delta, \theta)}{\partial \delta} p(\delta, \theta, t)\right)\right]
\end{aligned}
$$

where the potential includes effects due to the counterions, permanent dipole, induced, and intrinsic polarizability

$$
V(\delta, t)=\underbrace{n^{2} q^{2} \delta^{2} / 2 \alpha}_{\text {counterions }}-\underbrace{n q \delta E(t) \cos \theta}_{\text {induced }}-\underbrace{\mu_{p} E(t) \cos \theta}_{\text {permanent moment }}-\underbrace{\frac{\Delta \alpha E^{2}}{2} \cos ^{2} \theta}_{\text {intrinsic polarizability }}
$$

Here the anisotropy in polarizability is expressed as $\Delta \alpha=\alpha_{\|}-\alpha_{\perp}$. 
In the case when the polyion atmosphere is isotropic eq. (6.26) becomes

$$
\begin{aligned}
& \frac{\partial p(\delta, \theta, t)}{\partial t}=\frac{\Theta}{\sin \theta} \frac{\partial}{\partial \theta} \sin \theta \frac{\partial p(\delta, \theta, t)}{\partial \theta}+\Theta \beta q n E(t) \delta\left[2 \cos \theta p(\delta, \theta, t)+\Theta \sin \theta \frac{\partial p(\delta, \theta, t)}{\partial \theta}\right] \\
& \quad+D\left[\frac{\partial^{2} p(\delta, \theta, t)}{\partial \delta^{2}}+\frac{\beta n^{2} q^{2}}{\alpha} p(\delta, \theta, t)+\left(\frac{\beta n^{2} q^{2} \delta}{\alpha}-\beta n q E(t) \cos \theta\right) \frac{\partial p(\delta, \theta, t)}{\partial \delta}\right] .(6.28)
\end{aligned}
$$

Moment equations can be developed from eq. (6.26) into a hierarchy

$$
\begin{gathered}
\frac{d<\delta>}{d t}=-<\delta>/ \tau+D \beta n q E(t)<\cos \theta> \\
\frac{d<\delta^{2}>}{d t}=2 D-\frac{2<\delta^{2}>}{\tau}+2 \beta D n q E(t)<\delta \cos \theta>.
\end{gathered}
$$

The equations for the spatial and angular moments are

$$
\begin{aligned}
& \frac{d<\delta^{m}>}{d t}=m(m-1) D<\delta^{m-2}>-\frac{m<\delta^{m} \geq}{\tau}+2 \beta D n m q E(t)<\delta^{m-1} \cos \theta> \\
& \frac{d<\delta^{m} \cos ^{s} \theta>}{d t}=s(s-1)<\sin ^{3} \theta \cos ^{s-2} \theta \delta^{m}>-2 s<\sin \theta \cos ^{s} \theta \delta^{s}> \\
& +\Theta \beta n q E(t)<\sin \theta \cos ^{s+1} \theta \delta^{m+1}>+\Theta\left(2 \sin \theta \cos ^{s+1} \theta-s \sin ^{3} \theta \cos ^{s-1} \theta\right) \\
& \quad+m(m-1) D<\delta^{m-2} \cos ^{s} \theta>-\frac{m<\delta^{m} \cos ^{s} \theta>}{\tau} \\
& \quad+2 \beta D n m q E(t)<\delta^{m-1} \cos ^{s+1} \theta>.
\end{aligned}
$$

Equation (6.29) indicates that the time rate of change of the dipole moment is due to a relaxation term plus a driving term that is linear in electric field. This is similar to the Debye equation. Comparison to eq. (6.24) indicates that the effect of the electric field is to add a driving term to eq. (6.29).

The model developed in this section yields an equation of motion for the counterion sheath (eq. (6.29)) and polarization. In this model the polarization has a single relaxation time.

\section{Charge Transfer in DNA}

Szent-Gyorgyi concluded from experiments on proteins that biological materials have semiconducting properties. In the ensuing years a great deal of research has been 
performed on conduction in biopolymers. The base-pair sequences in DNA are aperiodic, whereas the phosphate backbone is periodic. Periodicity promotes semiconductivity. In addition, DNA contains counterions and bound water. This complicated structure has prevented the details of electronic conduction in DNA from being uncovered. Recently, a self-consistent field theory has been used for modeling biopolymers [74].

The semiconducting properties originate in internal charge-transfer mechanisms [2]. The semiconducting activation energies are on the order of 1 to $1.5 \mathrm{eV}$. The conduction is due to protonic and electronic activity. In the dry state, biopolymers exhibit conduction both by electrons and protons. Risser et al. [75] and Stemp [76] have studied electron transfer in single-stranded DNA and found it to be a good sensor of strand length with rigidly attached donors and receptors.

In DNA the periodic component of the base pairs produces band gaps. The aperiodic component of the base pairs acts as impurities in the band gap formed by the phosphate backbone. Typical valence and conduction-band gaps are in the order of 0.3 to $0.8 \mathrm{eV}$ and energy band gaps of around 10 to $12 \mathrm{eV}$. Bloch wavefunctions can be used for modeling since the system is approximately periodic.

In proteins, there are two main areas of charge transport. The charge transport is in the main polypeptide chains and through the hydrogen-bonded crosslinks. Recent research has concluded that conduction through the main polypeptide chains dominates over the hydrogen-bond mechanism [74]. Beratan et al. [77] proposed a model of electron tunneling in proteins with donor-acceptor interaction which is mediated by the covalent bonds between amino acids.

Electron transfer in DNA takes place primarily along the axis of the molecule in which there are four basic types. These include extra electrons, singlet and triplet $\pi$ type molecular excited states, and holes. The electrons in $\pi$-orbitals are delocalized and do not participate in bonding. They are free to move about the carbon nuclei in a molecule. On the other hand, $\sigma$-orbitals are symmetrical about the bond axis with localized $\mathrm{C}-\mathrm{C}$ and $\mathrm{C}-\mathrm{H}$ bonds. The electrons participating in these bonds are around the line joining the two nuclei and are localized. The bases in DNA possess an electronic $\pi$ system, exhibit electron delocalization, and have low-lying $\pi$-type orbitals where excited electrons may reside. [75,78-81] Since the bases are stacked close together, charge transfer can occur from base to base. Hanlon [82] studied protonation of DNA and found a higher density of protons in the minor groove than in the counterion solution 
surrounding the molecule.

The electron-transfer rate between donor and acceptor has been measured with photo-induced, excited state, and flash-quenching ground state techniques and with transient-absorption methods. The electron transfer rate is

$$
k=\nu \exp \left(-\left(\lambda-\Delta E_{m}\right)^{2} / 4 k_{B} \lambda T\right)
$$

where $T$ is temperature, $\lambda$ is reorganization energy, and $\Delta E_{m}$ is potential difference between sites.

The predicted decay length of charge transfer is $13 \mathrm{~nm}$ and typical electron chargetransfer rate is $10^{6} \mathrm{~s}^{-1}$. This large decay with distance makes sense since long-range transfer in DNA would not be advantageous from a molecular damage standpoint. For example, many proteins attached to the DNA would not function normally with large charge transfer taking place. Additionally, molecular damage becomes more probable during times of high charge transport [79].

The efficiency of DNA in allowing long-range electron transfer gives support to the notion that stacked aromatic heterocycles can serve as $\pi$-ways. The results of research in this area raise the possibility that DNA uses electron transfer in gene replication. Electromagnetic fields may be able to stimulate biosynthesis in cells, particularly in DNA. Electric fields surely interact with cell membranes, but recent evidence points to the possibility that magnetic fields can play a role in gene activation [83]. The process by which this could happen is through strong charge transport in DNA interacting with applied magnetic fields. Recent experiments on charge transport have demonstrated that current densities of at least $1 \times 10^{4} \mathrm{~A} / \mathrm{m}^{2}$ can be achieved in DNA. The localization of the gene segment through charge transport could be achieved either from bending or rolling of the base pairs or by enzyme interaction with base pairs in a segment. Electric fields could also play a role in gene activation through bending of DNA or formation of double layers affecting charge flow in the molecule.

There is evidence that hydration of DNA promotes conductivity and semiconductivity $[27,84]$. These studies found that relaxation of double-stranded DNA in an applied d.c. electric field of 1500 to $3000 \mathrm{~V} / \mathrm{cm}$ had a 10 percent increased conductivity. Intrinsic semi-conductivity in DNA increases exponentially with water content up to concentrations of 25 percent. Thereafter the effects are dominated by protonic activity. In the studies of Bonincontro [27], mixtures of $\mathrm{NaCl}$ and DNA from herring sperm of sizes of 
300 to 1000 nucleotide pairs were dissolved in water at a concentration of $2 \mathrm{mg} / \mathrm{ml}$. The samples were then dialyzed with solutions of $0.4 \mathrm{~mol}$ counterion solution ( $\mathrm{Na}$ and $\mathrm{Li}$ ) then lyophilized (freeze drying) and ground to a powder. For many biomolecules the conductivity follows a temperature dependence similar to inorganic semiconductors

$$
\sigma=\sigma_{0} \exp \left(-E / k_{B} T\right)
$$

\section{Measurements of DNA}

\subsection{Overview of Past Measurements}

Measurements on conducting liquids are complicated by electrode polarization [85] and by low-frequency dispersion (LFD) [86]. Electrode polarization is caused by the buildup of conducting ions on the capacitor plates producing an electrical double layer. Electrode polarization influences primarily the real part of the permittivity. Since the electrode capacitance is not a property of the material under test it must be removed from the measurement.

In the literature three relaxations have been reported, $\gamma, \delta$, and $\alpha$ as indicated in figure 3.2. The $\gamma$ relaxation occurs near $18 \mathrm{GHz}$ and has been ascribed to water. The $\delta$ relaxation is weak, occurs around $100 \mathrm{MHz}$, and is independent of molecular mass. This relaxation is due to the motion of condensed ions within a subunit of the DNA molecule $[37,87,88]$. The $\alpha$ relaxation occurs for DNA in the range 1 to $100 \mathrm{~Hz}$. The lowfrequency $\alpha$ relaxation depends on molecular mass and length and is due to fluctuations in the counterion. sheath.

Measurements on DNA in solution have been performed by many researchers over the years $[32,36,40]$. Good overviews of measurements of dielectric relaxation of DNA are given in Sorriso [5], Takashima [1], and Grant [88]. The measurements can be divided between those studies that measured DNA above $1 \mathrm{MHz}$ and those studies that measured at low frequencies $(\leq 1000 \mathrm{~Hz}$ ). Higher-frequency measurements were made by a number of authors $[40,89-92]$. Low-frequency measurements have been made by Takashima, Sakamoto, Hanss, and Tung $[16,20,32,93]$. In studies of the relaxation of DNA in saline solutions, Takashima observed an $\alpha$ relaxation at around $100 \mathrm{~Hz}[1,16]$, for DNA molecular masses of $5 \times 10^{6} \mathrm{D}$. Hanss and Sakamoto $[10,20,32,35,36,94]$ performed experiments on the same length molecules and found relaxations in the 5 to $1000 \mathrm{~Hz}$ range. Takashima used a capacitor with platinum-blacked electrodes to 
measure permittivity from $1 \mathrm{~Hz}$ to $1000 \mathrm{~Hz}$. Sakamoto used a four-terminal device and a conductivity standard to eliminate the effects of electrode polarization and d.c. conductivity [32]. He measured DNA of molecular mass $4 \times 10^{6} \mathrm{D}$ from $0.2 \mathrm{~Hz}$ to 30 $\mathrm{kHz}$ and found a relaxation near $5 \mathrm{~Hz}$. The conductivity of their solution was from $2000 \mu \mathrm{S}$ to $13,000 \mu \mathrm{S}$. Hanss and Bernengo [20] found a peak at 1-10 Hz. Tung [40] and Takashima [16] obtained a decrease in dielectric increment for calf-thymus DNA as concentration increased, whereas Sakamoto found the reverse trend [10]. Sakamoto [10] found that concentration dependence of the DNA became important around 0.02 percent and above. Sakamoto attributes this to intermolecular interaction. In figure 8.1, a ColeCole plot of dielectric increment is plotted from Sakamoto $[10,32,35,36]$.

\subsection{Permittivity Measurements and Electrode Polarization}

The permittivity is can be written in the form

$$
\epsilon^{*}=\epsilon_{0}\left[\epsilon_{r}^{\prime}-j\left(\epsilon_{r}^{\prime \prime}+\frac{\sigma}{\omega}\right)\right]
$$

At low frequencies, a number of different dispersive processes occur. Direct current conductivity where $\sigma \propto 1 / \omega$ produces a large dispersion in $\epsilon^{\prime \prime}$ as $\omega \rightarrow 0$. In this phenomena the real part of the permittivity approaches a constant value, whereas the imaginary part increases as $1 / \omega$. Another phenomenon is low-frequency dispersion where both the real and imaginary parts of the permittivity vary as $\omega^{n-1}$ where $n$ is a positive number slightly greater than 0 , for example $n=0.1$. Maxwell-Wagner circuit models have been used successfully to model the low-frequency behavior of highly conductive materials.

In highly conductive solutions the interaction of the electrodes with mobile charge can mask the real part of the permittivity through electrode polarization. The electrodepolarization effect can be modeled by lumped circuit elements [16]

$$
\begin{gathered}
C=C_{s}+\frac{1}{\omega^{2} R^{2} C_{p}}, \\
R=R_{s}+R_{p}+R_{s} \omega^{2} R^{2} C^{2},
\end{gathered}
$$

where $C, R$ are the measured capacitance and resistance, $C_{p}, R_{p}$ are the electrode double-layer capacitance and resistance, and $C_{s}, R_{s}$ are the sample capacitance and resistance. 

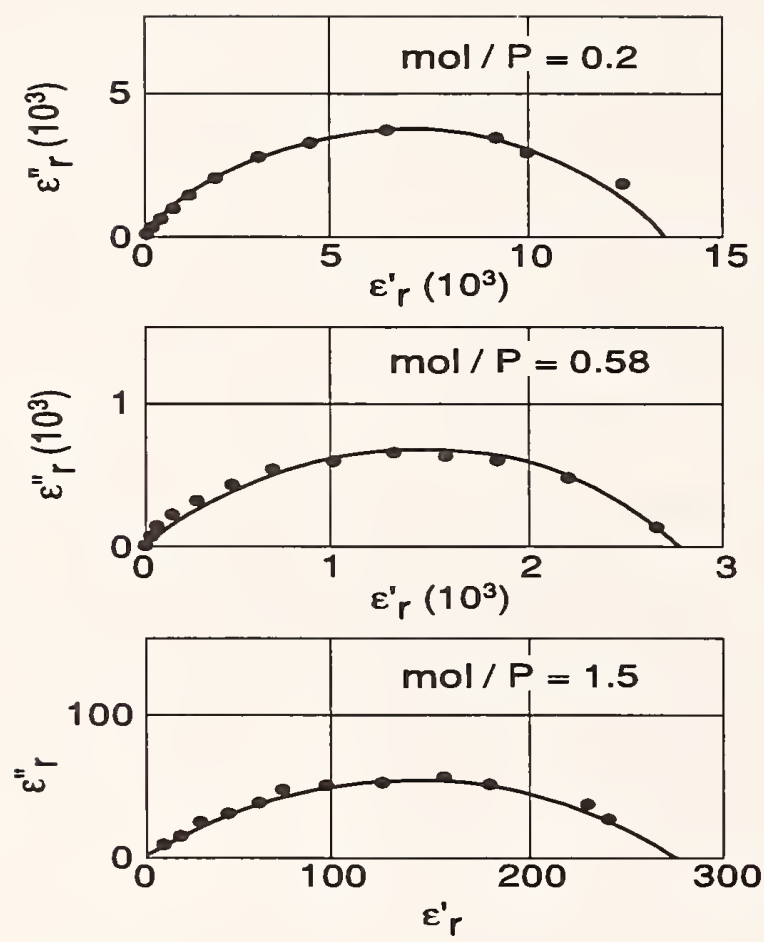

Figure 8.1. Dielectric increment of calf thymus for various molecular masses and phosphate residues in Cole-Cole format [36]. 


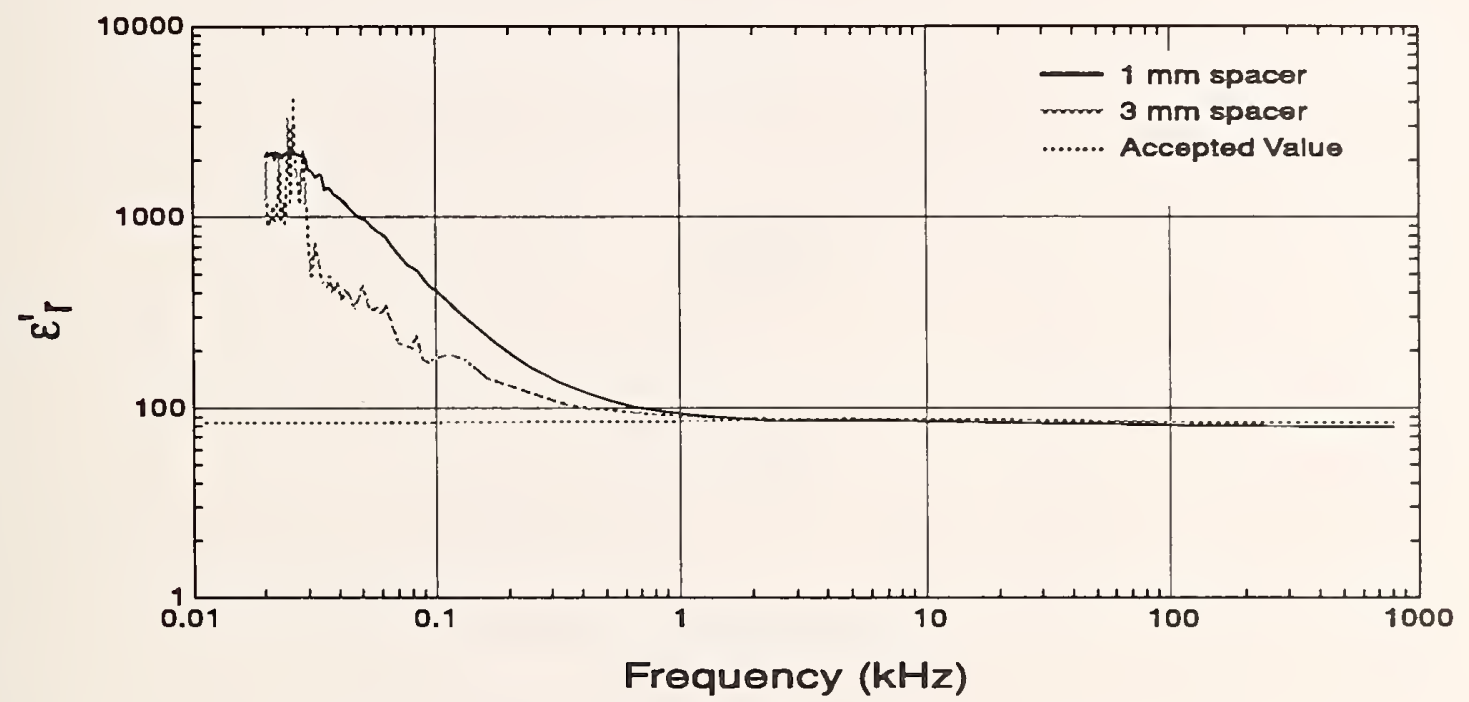

Figure 8.2. Capacitor measurements of saline solutions of two different thicknesses.

A way to partially eliminate electrode polarization is by measuring capacitance and resistance for two different electrode separations (see figure 8.2). It is then assumed that $C_{p}$ is the same for each measurement and $C_{s}$ is then determined. The two-separation technique works well only in cases where the electrode-polarization effect is small. Another way of minimizing the electrode-polarization effect is to coat the capacitor plates with platinum black [95]. This lessens the influence of electrode polarization by decreasing the second term on the right side of eq. (8.2). The best approach is to bypass the electrode-polarization problem and use a four-probe capacitor system. 


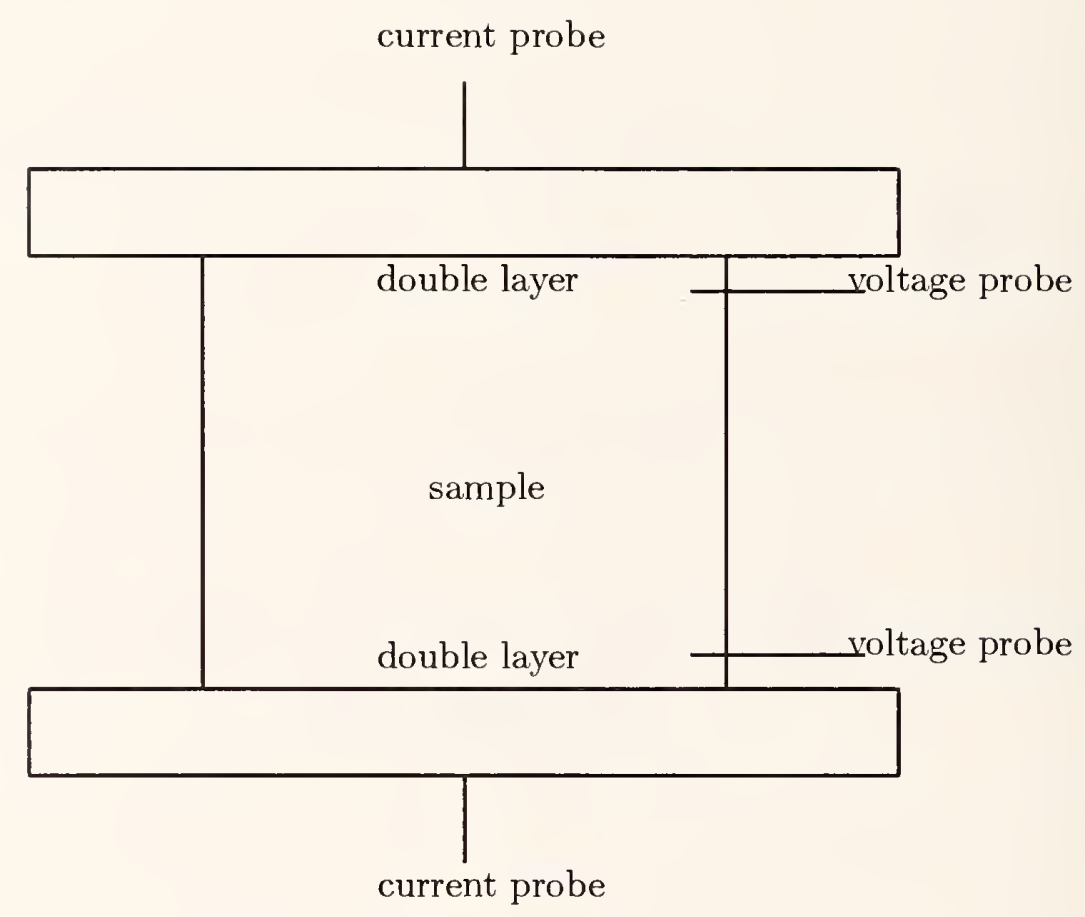

Figure 8.3. Capacitor with sample for minimizing electrode polarization. 


\subsection{Measurement Methods}

\subsubsection{Capacitive Measurement Techniques}

The capacitor is useful when the electromagnetic wavelength in the material is much longer than the separation between the plates. In this measurement the sample is assumed to be immersed in a strong electric field and weak magnetic field. A difficulty with capacitor measurements resides in minimizing effects of the fringing field and electrode polarization. The fringe field is usually partially eliminated by using guards. The capacitance for a parallel plate of separation $d$ and area $A$, with no fringing of fields near edges is given by

$$
C=\frac{\epsilon^{\prime} A}{d}
$$

The conductance at low frequency is given by

$$
G=\omega \frac{\epsilon^{\prime \prime} A}{d}
$$

The permittivity can be obtained from measurements of $C$ and $G$ and is given by

$$
\epsilon_{r}^{*}=\frac{C-j G / \omega}{C_{a i r}-j G_{a i r} / \omega}
$$

The $*$ indicates the permittivity is complex.

The effects of electrode polarization when measuring conducting liquids at low frequencies must be addressed.

\subsubsection{Four-Probe Technique}

The main problem with capacitance techniques for highly conductive liquids is electrode polarization. The four-probe capacitance technique overcomes electrode problems [96, 97] by measuring the voltage drop away from the plates and thereby avoiding the double layer (see figure 8.3).

The permittivity is calculated for this measurement by

$$
\begin{aligned}
& \Delta Z^{\prime}=-\frac{1}{C_{0}}\left(\frac{\epsilon_{0}}{\sigma}\right)^{2} \Delta\left(\omega \epsilon^{\prime \prime}\right), \\
& \Delta Z^{\prime \prime}=-\frac{1}{C_{0}}\left(\frac{\epsilon_{0}}{\sigma}\right)^{2} \Delta\left(\omega \epsilon^{\prime}\right),
\end{aligned}
$$




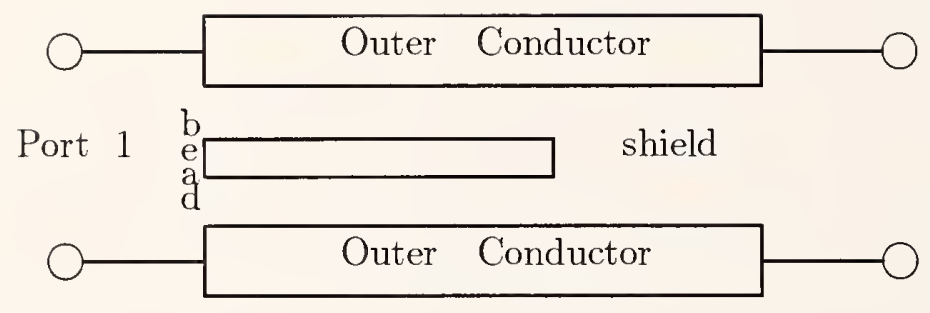

Figure 8.4. A shielded open circuit termination. The inner and outer radii of conductors are $a$ and $b$.

where $\omega \Delta \epsilon^{\prime \prime}$ is the difference in loss times angular frequency between solvent and solute and $C_{0}$ is the vacuum capacitance of the cell. $\Delta Z^{\prime}$ and $\Delta Z^{\prime \prime}$ are the measured differences in real and imaginary parts of the impedance between solvent and solute.

\subsubsection{The Open-Circuited Holder for Liquid Measurement}

The shielded open-circuited coaxial line sample holder has been used for years (see figure 8.4) for dielectric measurements of liquids and powders in the microwave band [98]. This fixture is composed of section of coaxial line where the outer conductor extends beyond the end of the inner conductor. The advantage of an open-circuited holder is the ease of sample installation, the broad frequency capability, and the strong electric field in the sample region. These holders can be used for measurements from $1000 \mathrm{~Hz}$ to $20 \mathrm{GHz}$. The sample holder operation is based on an accurate model of a coaxial line terminated in a shielded open circuit. The model we use is based on a full-wave solution. The fullmode model is more accurate than the commonly used capacitance expansion [99, 100].

The sample holder has been found useful in both ambient and high-temperature measurements. Von Hippel [101] used an open-circuited sample holder for liquid measurements. Bussey [99] extended the open-circuited holder technique to higher frequencies with an admittance model. Scott [102] studied the instabilities encountered in solving the relevant nonlinear open-circuit equations. Hill [103] studied in situ measurements of soils using open-circuited transmission lines. Jesch [104] used the shielded opencircuited holder for measurements on shale oil. Biological tissues have been measured 
using the shielded open-circuited line, for example by Stuchly and Stuchly [105].

\subsection{Dielectric Measurements on DNA in Solution}

We measured herring sperm DNA in mixtures of deionized water, protamine, and base solution from $20 \mathrm{~Hz}$ to $200 \mathrm{MHz}$. Our measurements were accomplished by use of a capacitor fixture from $20 \mathrm{~Hz}$ to $1 \mathrm{MHz}$ and an open-circuited holder from $300 \mathrm{kHz}$ to 200 $\mathrm{MHz}$. We used two capacitors. The first was nickel plated; the second was platinum blacked to minimize effects from the electrode. We experienced a large decrease in electrode effects in the platinum-blacked fixture over the nickel-plated fixture. Typical capacitor measurements of DNA solutions are given in figures 8.5 through 8.10. To denature the DNA, samples were heated to $97{ }^{\circ} \mathrm{C}$ for $5 \mathrm{~min}$.; then the samples were placed on ice until cooled below room temperature. The samples were then allowed to come back to equilibrium at $22{ }^{\circ} \mathrm{C}$ and remeasured. The measurement was taken immediately after putting it in the fixture. The mass of the sample was measured before and after heating. The mass loss was less than 0.2 percent. In figures 8.5 and 8.6, the permittivities of DNA solutions as a function of protamine concentration are displayed. In figures 8.7 through 8.10, the permittivities of DNA solutions of both single (SS) and double-stranded (DS) DNA as a function of protamine concentration are displayed on a $\log$ - $\log$ scale. In figures 8.11 and 8.12 , results for measurements using the shielded open- circuited holder from $300 \mathrm{kHz}$ to $200 \mathrm{MHz}$ are shown.

At low frequency our results show strong electrode polarization and low-frequency dispersion. The static values of the real part of the permittivity in figures (8.7) and (8.9) can be estimated by drawing a straight line from the data in the $10 \mathrm{kHz}-100$ $\mathrm{kHz}$ range to $0 \mathrm{~Hz}$. At very low frequencies (less than $100 \mathrm{~Hz}$ ) we observed a drift in capacitance with time. We consistently see higher loss and higher permittivity in the denatured solutions than in the double-stranded solutions. These trends were also seen by Georgakilas [106]. We postulate that this is due in part to the increased conductivity in the single-stranded solutions. As the strands separate, counterions are released and thereby the conductivity increases. We think that the increase of the real part of the permittivity is due to increased electrode polarization as the conductivity increases. There are significant differences between the permittivity of the solvent and solutions containing DNA.

The observed polarization can be interpreted as due to motion of the counterion 


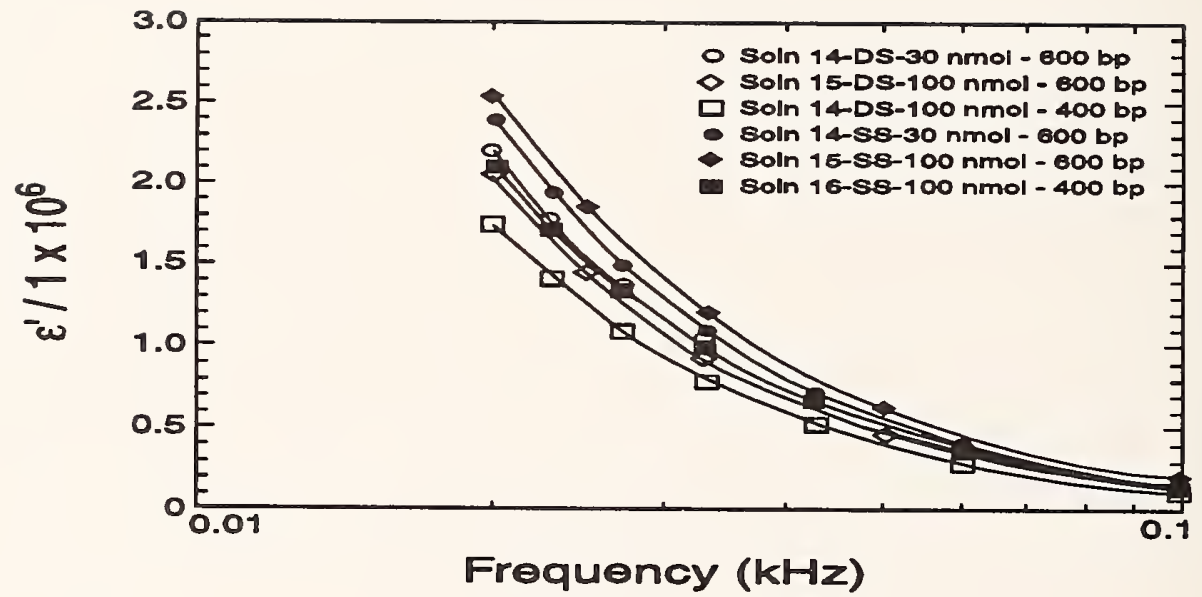

Figure 8.5. Measurements of the real parts of the permittivity of double-stranded and denatured DNA as a function of frequency. The amount of protamine cation was 30 nmol and 100nmol. Note TBE refers to a solution of tris, boric acid, and EDTA. Also (BP) stands for base pairs. 


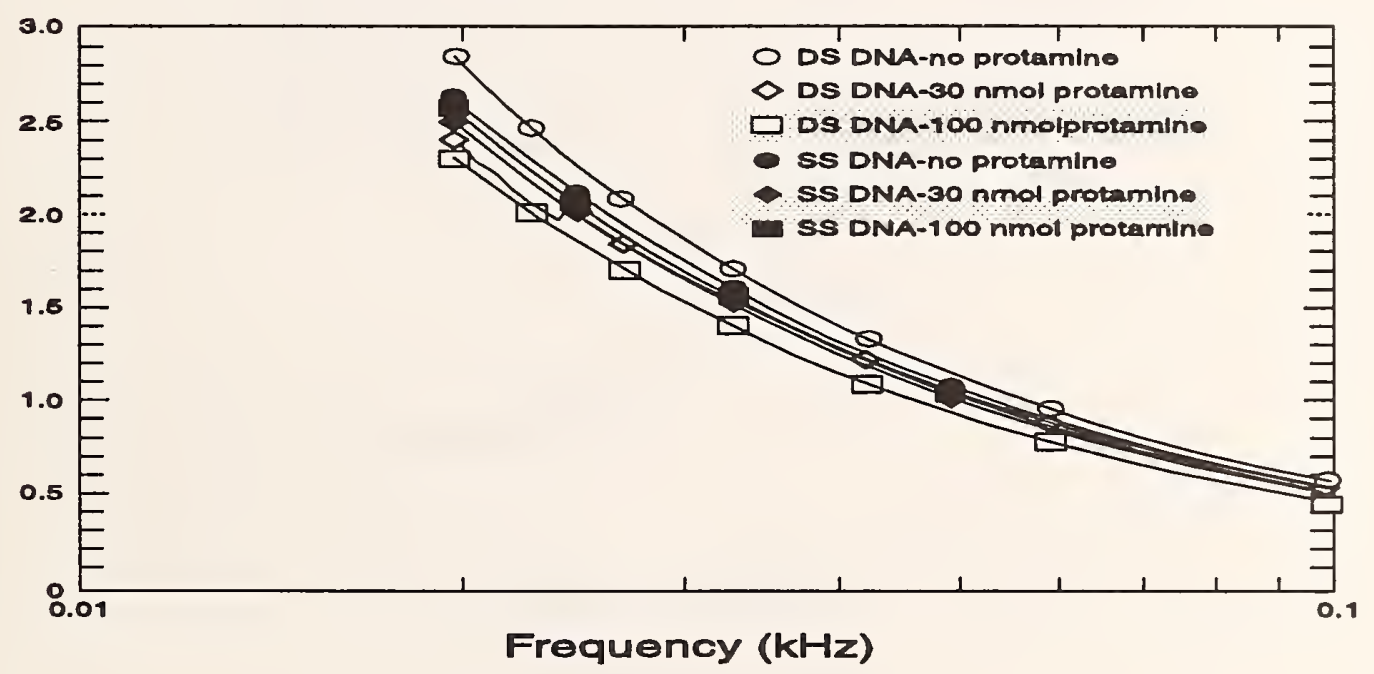

Figure 8.6. Capacitance measurements of the imaginary parts of the permittivity of double-stranded (DS) and denatured (SS) DNA as a function of frequency. The amount of protamine cation was $30 \mathrm{nmol}$ and $100 \mathrm{nmol}$. 


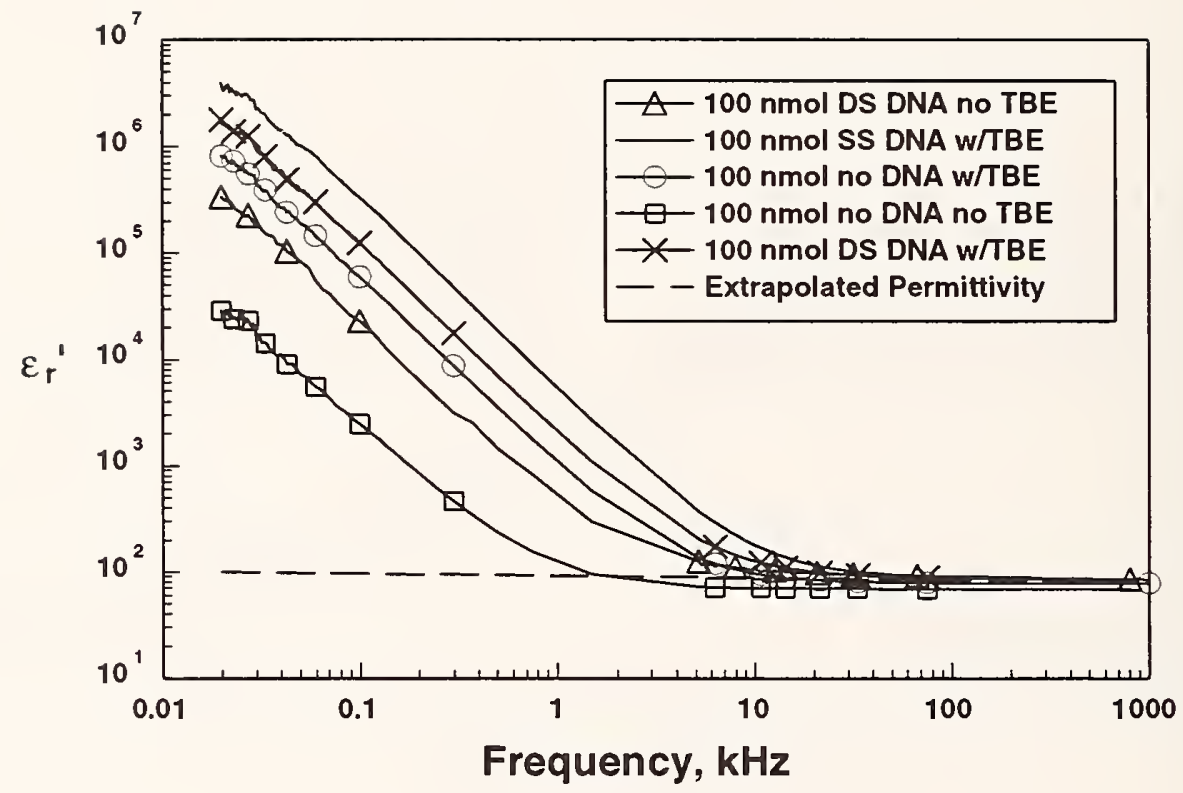

Figure 8.7. Capacitance measurements of the real parts of the permittivity of doublestranded (DS) and denatured (SS) DNA as a function of frequency on a log-log scale. The amount of protamine cation was $100 \mathrm{nmol}$ in TBE solvent. The estimated permittivity without electrode polarization is shown by the dashed curve. 


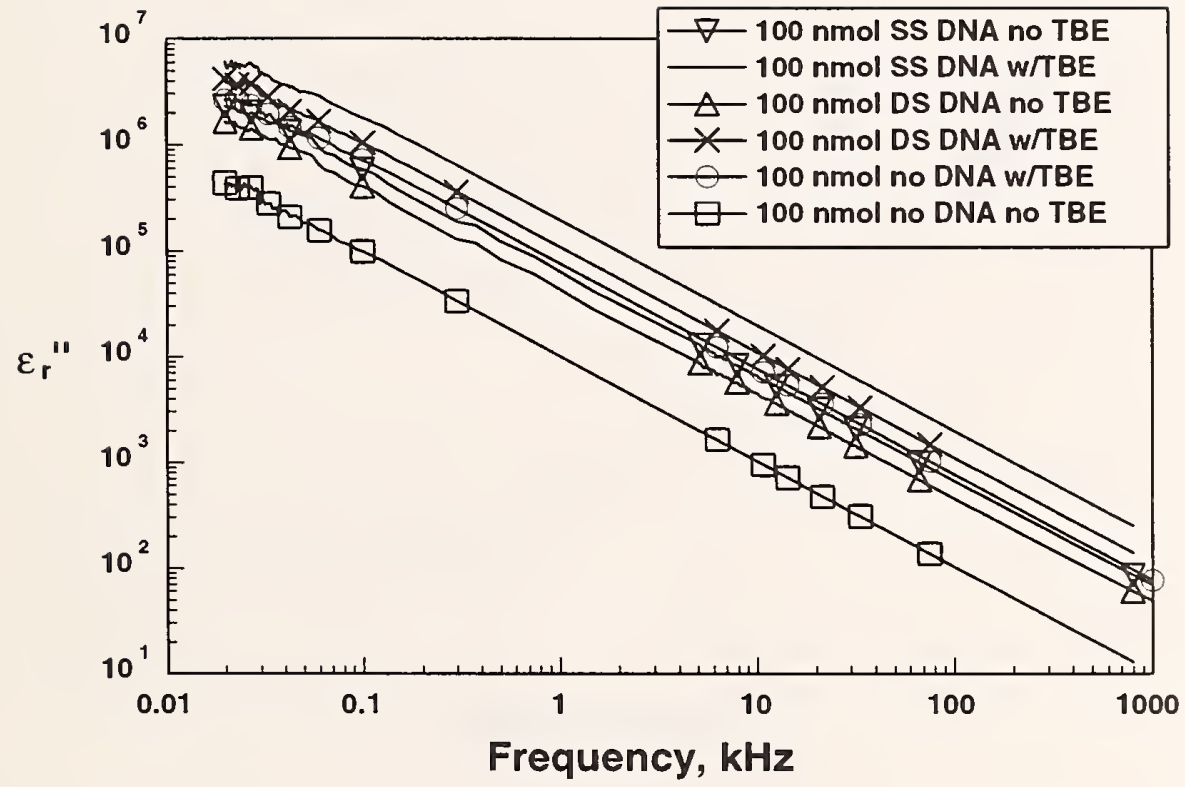

Figure 8.8. Capacitance measurements of the imaginary parts of the permittivity of double-stranded (DS) and denatured (SS) DNA as a function of frequency on a log-log scale in TBE solvent. The amount of protamine cation was $100 \mathrm{nmol}$. 


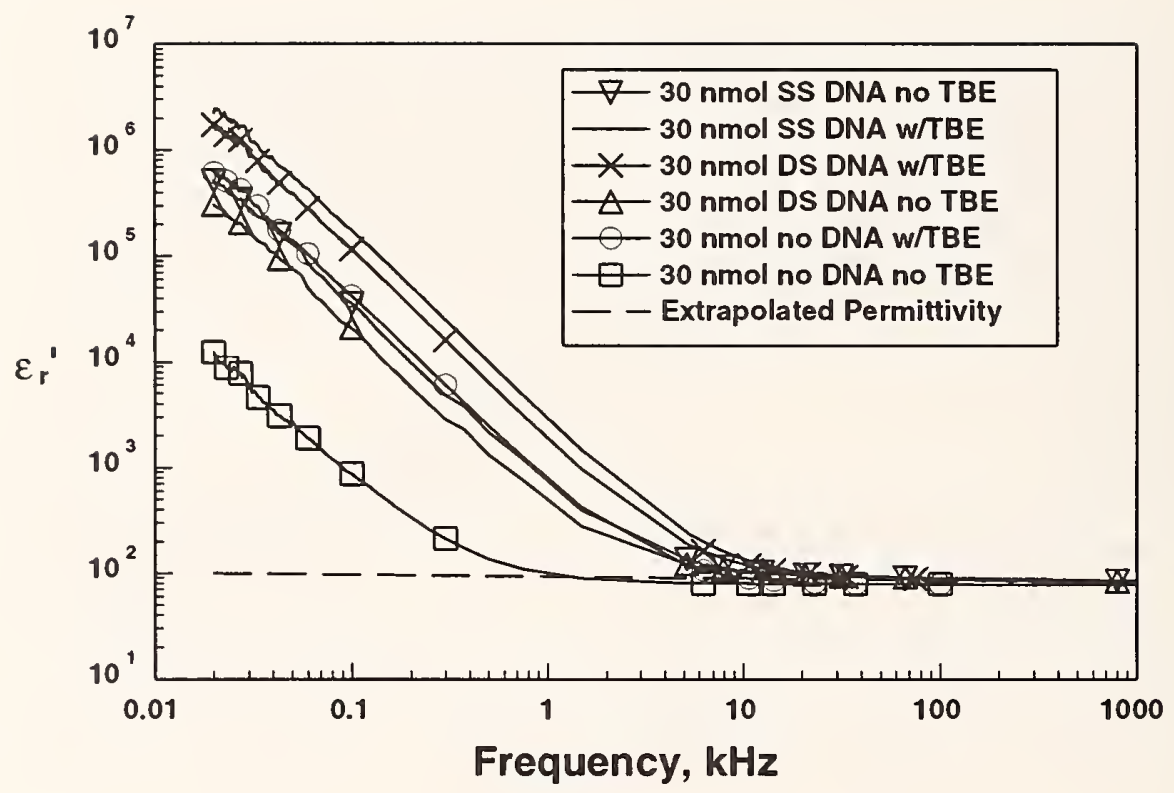

Figure 8.9. Capacitance measurements of the real parts of the permittivity of doublestranded (DS) and denatured (SS) DNA as a function of frequency on a log-log scale in TBE solvent. The amount of protamine cation was $30 \mathrm{nmol}$. The estimated permittivity without electrode polarization is shown by the dashed curve. 


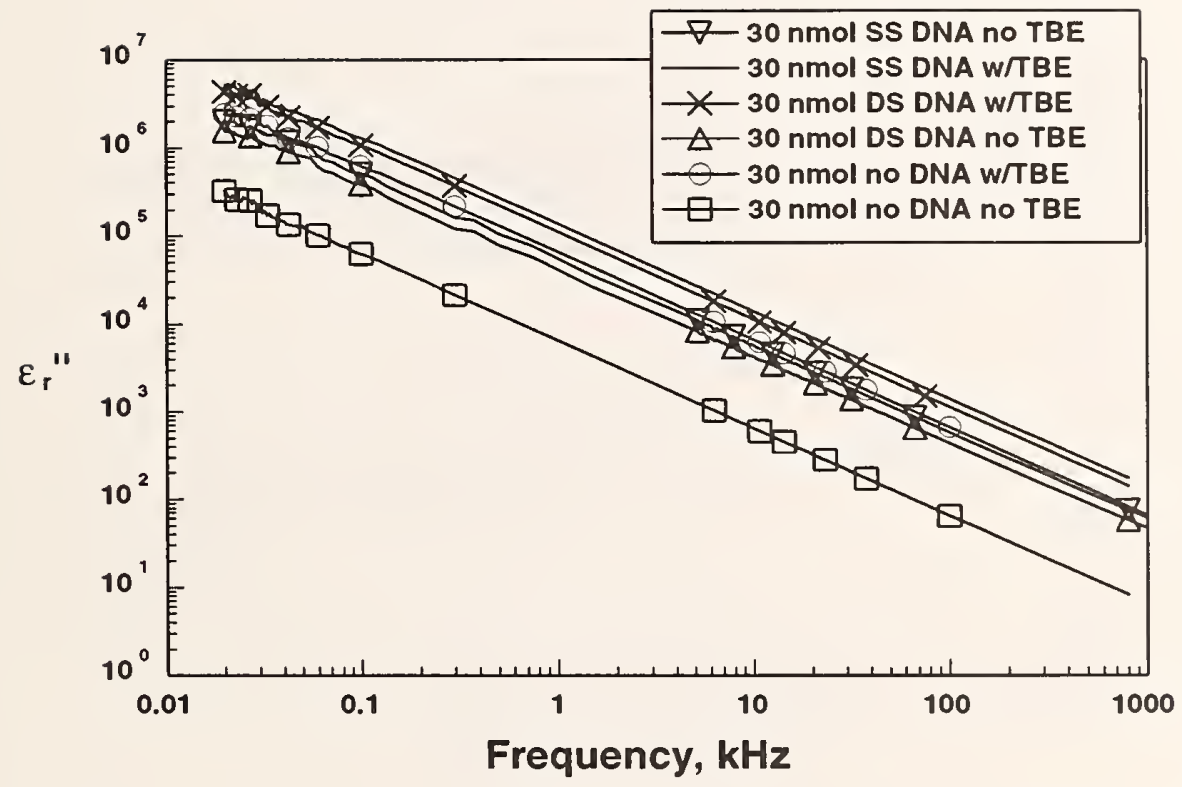

Figure 8.10. Capacitance measurements of the imaginary parts of the permittivity of double-stranded (DS) and denatured (SS) DNA as a function of frequency on a log-log scale in TBE solvent. The amount of protamine cation was $30 \mathrm{nmol}$. 


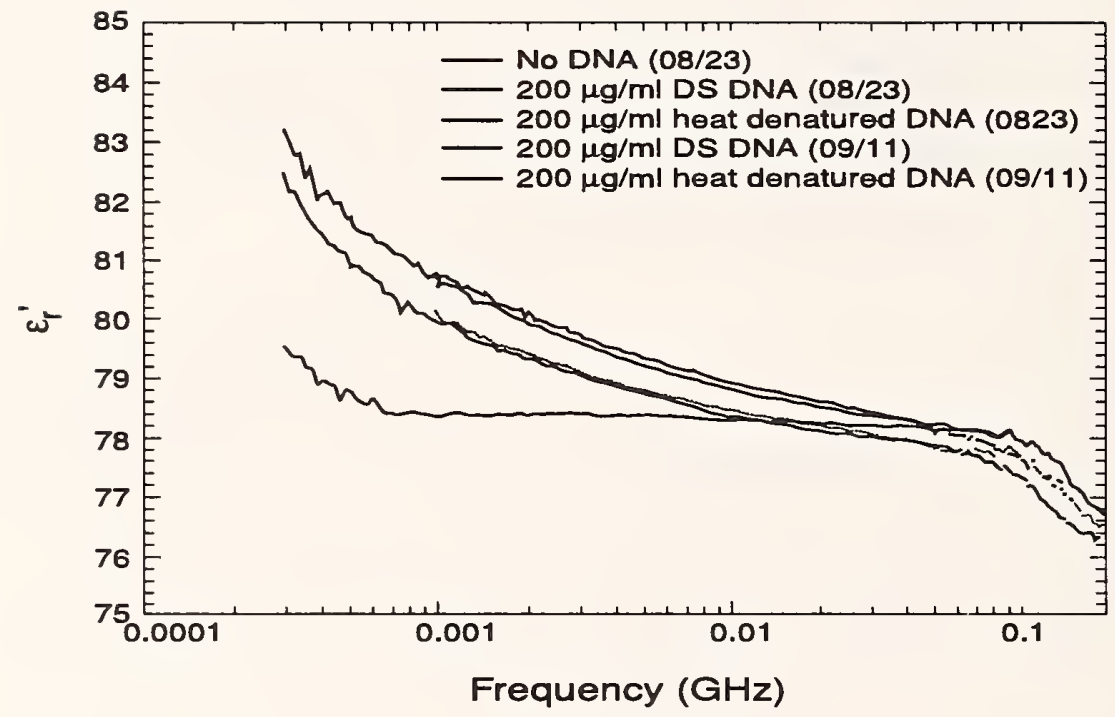

Figure 8.11. Shielded open-circuit holder measurements of the real parts of the permittivity of double-stranded DNA as a function of frequency on a log-log scale. 


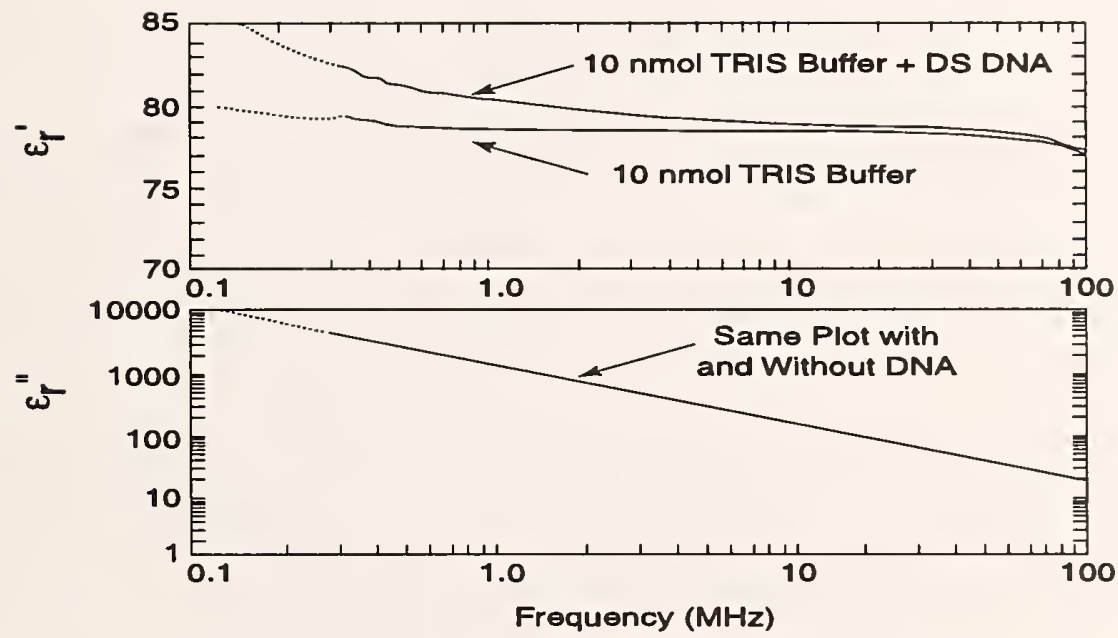

Figure 8.12. Shielded open-circuit holder measurements of the permittivity of doublestranded and denatured DNA as a function of frequency. 
sheath around the DNA molecule. This counterion effect is an induced polarization with the induced dipole moment parallel to the sheath major axis. The dispersion peak is masked by the strong electrode polarization and low-frequency dispersion.

Denaturation appears to proceed in stages [106]. First counterions are expelled from the molecule, which causes dissociation and strong phosphate-phosphate repulsion between strands. This increases the conductivity of the solution. As temperature is increased further the repulsive forces cause hydrogen bond rupture and denaturation.

\section{Discussion and Conclusions}

In this Technical Note we have overviewed dielectric relaxation of DNA in solution. We also studied the current understanding of the polarization of the DNA molecule. This required an understanding of counterion dynamics around the molecule and polarization mechanisms. To this end we overviewed polarization theory for polymer systems and developed an extension of the Poisson-Boltzmann equations for the electrode-DNA interface. We also developed a statistical-mechanical model of DNA in an ion atmosphere. We then studied past measurements of DNA and finally presented results of our measurements.

The low-frequency polarization observed in DNA is due to motion of the counterion sheath. This polarization increases as the square of the length of the molecule and decreases as temperature increases. An understanding of both the length and temperature dependence can be understood by models of the interaction of the counterion atmosphere with, the phosphate backbone of the molecule. Models that represent DNA as a string of rigid rods predict the correct temperature and length-dependent behavior.

In our measurements the dispersion peak is masked by the strong electrode-polarization and low-frequency dispersion. We observed higher loss and higher permittivity in the single-stranded solutions than in the double-stranded solutions. These observations were also made by Georgakilas [106]. We think that this is due in part to the increased conductivity in the single-stranded solutions. The increase of the real part of the permittivity we believe is due to increased electrode polarization as the conductivity increases.

We see evidence from conductivity measurements for the following sequence of stages in denaturation [106]. First counterions are expelled from the molecule which causes 
dissociation and strong phosphate-phosphate repulsion between strands. This increases the conductivity of the solution. As temperature is increased further the repulsive forces cause hydrogen-bond rupture and denaturation.

We would like to thank Albert Young and Mark Hollis of MIT Lincoln Laboratory, Stan Abramowitz of the Advanced Technology Program (ATP), members of the Genosensor Consortium, Eleanor Livingston for discussions and editing, and Claude Weil, John Grosvenor, and Michael Janezic for measurement and design assistance.

\section{References}

[1] Takashima, S. Electrical Properties of Biopolymers and Membranes. New York: Springer-Verlag; 1989.

[2] Pethig, R. Dielectric and Electronic Properties of Biological Materials. New York: John Wiley and Sons; 1979.

[3] Frank-Kamenetskii, M.D.; Anshelevich, V.V.; Lukashin, A.V. Polyelectrolyte model of DNA. Biopolymers 30: 317-330; 1987.

[4] Foster, K.R.; Saur, F.A.; Schwan, H.P. Electrorotation and levitation of cells and colloidal particles. Biophys. J. 63: 180-190; 1992.

[5] Sorriso, S.; Surowiec, A. Molecular Dynamics Investigations of DNA by Dielectric Relaxation Measurements; pages 259-279. 1982.

[6] Gueron, M.; Demaret, J.P. A simple explanation of the electrostatics of the B to Z transition. Biophys. 89: 5740-5743; 1992.

[7] Gulbrand, L.E.; Forrester, T.R.; Lynden-Bell, R.M. Distribution and dynamics of mobile ions in systems of ordered B-DNA. Molecular Phys. 67: 473-93; 1989.

[8] Pack, G.R.; Garrett, G.A.; Wong, L.; Lamm, G. The effect of a variable dielectric coefficient and finite ion size on Poisson-Boltzmann calculations of DNAelectrolyte systems. Biophys. J. 65: 1363-1370; 1993. 
[9] Saxena, V.K.; Zandt, L.L.Van. Effect of counterions on the spectrum of dissolved DNA polymers. Phys. Rev. A 45: 7610-7620; 1992.

[10] Sakamoto, M.; Hayakawa, R.; Wada, Y. Dielectric relaxation of DNA solutions. III. Effects of DNA concentration, protein contamination, and mixed solvents. Biopolymers 18: 2769-2782; 1979.

[11] Oncley, J.L. Proteins, Amino Acids and Peptides: Reinhold. 1943.

[12] Kirkwood, J.L.; Shumaker, J.B. The influences of dipole moment fluctuations on the dielectric increment of proteins in solution. Proc. Natl. Acad. Sci. USA 35: $855-862 ; 1952$.

[13] Maleyev, V.Y.; Semenov, M.A.; Gasan, A.I.; Kashpur, V.A. Physical properties of the DNA-water system. Biophys. 38: 785-801; 1993.

[14] Nanda, N.; Bagchi, B. Anomalous dielectric relaxation of aqueous protein solutions. J. Phys. Chem. A 102: 8217-8221; 1998.

[15] Renugopalakrishnan, V.; Lakshminarayanan, A.V.; Sasisekharan, V. Stereochemistry of nucleic acids and polynucleotides III. Electric charge distribution. Biopolymers 10: 1159; 1971.

[16] Takashima, S. Dielectric dispersion of deoxyribonucleic acid. J. Phys. Chem. 70(5): 1372-1379; 1966.

[17] Hogan, M.; Dattagupta, N.; Crothers, D.M. Transient electric dichroism of rodlike DNA molecules. Proc. Natl. Acad. Sci. 75: 195-199; 1978.

[18] Sakamoto, M.; Fujikado, T.; Hayakawa, R.; Wada, Y. Low frequency dielectric relaxation and light scattering in AC electric field of DNA solutions. Biophys. Chem. 11: 309-316; 1980.

[19] Oosawa, F. Counterion fluctuation and dielectric dispersion in linear polyelectrolytes. Biopolymers 9: 677-688; 1970.

[20] Hanss, M.; Bernengo, J.C. Dielectric relaxation and orientation of DNA molecules. Biopolymers 12: 2151-2159; 1973. 
[21] Plum, G.E.; Bloomfield, V.A. Contribution of asymmetric ligand binding to the apparent permanent dipole moment of DNA. Biopolymers 29: 1137-1146; 1990.

[22] Crothers, D.M. Upsetting the balance of forces in DNA. Science 266: 1819-1820; 1994 December.

[23] Strauss, J.K.; Maher, J. DNA bending by asymmetric phosphate neutralization. Science 266: 1829-1834; 1994.

[24] Antosiewicz, J.; Porschke, D. An unusual electrooptical effect observed for DNA fragments and its apparent relation to a permanent electric moment associated with bent DNA. Biophysical Chemistry 33: 19-30; 1989.

[25] Lamm, G.; Pack, G.R. Local dielectric constants and Poisson-Boltzmann calculations of DNA counterion distributions. Int. J. Quant. Chem. 65: 1087-1093; 1997.

[26] Jayaram, B.; Sharp, K.A.; Honig, B. The electrostatic potential of B-DNA. Biopolymers 28: 975-993; 1989.

[27] Bonincontro, A.; Careri, G.; Giansanti, A.; Pedone, F. Water induced conductivity of DNA: A dielectric- gravimetric study. Phys. Rev. A 38(12): 6446-6447; 1988 December.

[28] Ise, N.; Eigen, M.; Schwarz, G. The orientation and dissociation field effect of DNA in solution. Biopolymers 1: 343-352; 1963.

[29] Bur, A.J.; Roberts, D.E. Rod and random-coil behavior of poly(n-butyl isocyanate) in dilute solution. J. Chem. Phys. 51: 406-420; 1969 April.

[30] Kirkwood, J.G. The visco-elastic properties of solutions of rod-like macromolecules. J. Chem. Phys. 44: 281-283; 1951 March.

[31] Yu, H.; Bur, A.J.; Fetters, L.J. Rodlike behavior of poly(n-butyl isocyanate) from dielectric measurements. J. Chem. Phys. 44: 2568-2576; 1966 November.

[32] Sakamoto, M.; Kanda, H.; Hayakawa, R.; Wada, Y. Dielectric relaxation of DNA in aqueous solutions. Biochem. 15: 879-892; 1976. 
[33] Edwards, G.; Hochberg, D.; Kephart, T.W. Structure in the electric potential emanating from DNA. Phys. Rev. E 50: R698-R701; 1994.

[34] Hochberg, D.; Kephart, T.W.; Edwards, G. Structure information in the local electric field of dissolved B-DNA. Phys. Rev. E 49: R851-R867; 1994.

[35] Sakamoto, M.; Hayakawa, R.; Wada, Y. Dielectric relaxation of DNA solutions. II. Biopolymers 17: 1507-1512; 1978.

[36] Sakamoto, M.; Hayakawa, R.; Wada, Y. Dielectric relaxation of DNA solutions. IV. Effects of salts and dyes. Biopolymers 19: 1039-1047; 1980.

[37] Bonincontro, A.; Caneva, R.; Pedone, F. Dielectric relaxation at radio frequencies of DNA- protamine systems. J. Non-crystalline Solids 131-133: 1186-1189; 1991.

[38] Duguid, J.G.; Bloomfield, V.A. Electrostatic effects on the stability of condensed DNA in the presence of divalent cations. Biophys. J. 70: 2838-2846; 1996.

[39] Mctague, J.P.; Gibbs, J.P. Polyions. J. Chem. Phys. 44: 4295-4301; 1967.

[40] Tung, M.S.; Molinari, R.J.; Cole, R.H.; Gibbs, J.H. Influence of temperature and ionic strength on the low-frequency dielectric dispersion of DNA solutions. Biopolymer 16: 2653; 1977.

[41] Takashima, S. Dielectric study of polynucleotides. Acta Physiol. Scand. Suppl. 10: $32 ; 1945$.

[42] Ando, T.; Yamasaki, M.; Suzuki, K. Protamines. New York: Springer-Verlag; 1973.

[43] Yu, S.S.; Li, H.J. Helix-coil transition and conformational studies of protamineDNA complexes. Biopolymers 12: 2777-2788; 1973.

[44] Inoue, S.; Ando, T. Interaction of clupeine with deoxyribonucleic acid I: Thermal melting and sedimentation studies. Biochem. 9: 388-394; 1970.

[45] Bonincontro, A.; Caneva, R.; Pedone, F.; Romano, T.F. Complex dielectric constant of arginine-DNA and protamine-DNA aqueous systems at $10 \mathrm{GHz}$. Phys. Med. Biol. 34: 609-616; 1989. 
[46] Galindo, C.E.; Sokoloff, J.B. Uncoiling transition for DNA in solution. Phys. Rev. E 54: 691-705; 1996.

[47] Lawley, P.D. Helix-coil transition. Biophys. Acta 21: 481; 1956.

[48] Soumpasis, D.M. Helix-coil transition in DNA. Proc. Natl. Acad. Sci. 81: 5116; 1984.

[49] Friedman, R.A.; Honig, B. A free energy analysis of nucleic acid base stacking in aqueous solution. Biophys. J. 69: 1528-1535; 1995.

[50] Cantor, C.R.; Schimmel, P.R. Biophysical Chemistry: Part III: W. H. Freeman. 1971.

[51] Yakushevich, L.V. Nonlinear DNA dynamics: a new model. Phys. Lett. A 136: 413-417; 1989.

[52] Muto, V.; Lomdahl, P.S.; Christiansen, P.L. Two-dimensional discrete model for DNA dynamics: Longitudinal wave propagation and denaturation. Phys. Rev. A 42: 7452-7458; 1990.

[53] Muto, V. Local denaturation in DNA molecules. J. Biol. Phys. 19: 113-122; 1993.

[54] Peyrard, M.; Bishop, A.R. Statistical mechanics of a nonlinear model for DNA denaturation. Phys. Rev. Lett. 62: 2755-2758; 1989.

[55] Prabhu, V.V.; Young, L.; Prohofsky, E.W. Hydrogen-bond melting in B-DNA copolymers in a mean-field self-consistent phonon approach. Phys. Rev. B 39: $5436-5443 ; 1989$.

[56] Mandel, M.; Odijk, U. Dielectric properties of polyelectrolyte solutions. In Ann. Rev. Phys. Chem.; pages 75-108. Annual Reviews, Inc.; 1984.

[57] Touw, F.V.der; Mandel, M. Dielectric increment and dielectric dispersion of solutions containing simple charged linear macromolecules. Biophys. Chem. 2: 218$241 ; 1974$.

[58] Honig, B.; Nicholls, A. Classical electrostatics in biology and chemistry. Science 268: 1144-1149; 1995. 
[59] Oosawa, F. Polyelectrolytes: Marcel Dekker, Inc. 1971.

[60] Zakrzewska, K.; Madami, A.; Lavery, R. Poisson-Boltzmann calculations for nucleic acids and nucleic acids complexes. J. Chem. Phys. 204: 263-269; 1996.

[61] Grosse, C. Microwave absorption of suspensions of DNA type particles in electrolyte solution. Alta Frequenza 58: 365-368; 1989.

[62] Dukhin, S.S.; Shilov, V.N. Dielectric Phenomena and the Double layer in Disperse Systems and Polyelectrolytes: John Wiley and Sons. 1973.

[63] Brett, C. M.A.; Brett, A. M.O. Electrochemistry: Oxford Science Publications. 1993.

[64] Saxena, V.K.; Zandt, L.L.Van; Schroll, W.K. Effective field approach for longrange dissolved DNA polymer dynamics. Phys. Rev. A 39: 1474-1481; 1988.

[65] Zandt, L.L.Van. Resonant microwave absorption by dissolved DNA. Phys. Rev. Lett. 57: 2085-2087; 1986.

[66] Saxena, V.K.; Dorfmann, B.H.; Zandt, L. L.V. Identifying and interpreting spectral features of dissolved poly (dA)-poly $(\mathrm{dT})$ DNA polymer in the high-microwave range. Phys. Rev. A 43: 4510-4516; 1991.

[67] Yumakawa, H. Modern theory of polymer solutions.: Harper and Row. 1971.

[68] Szabo, A.; Haleem, M.; Eden, D. Theory of the transient electric birefringence of rod-like polyions: coupling of rotational and counterion dynamics. J. Chem. Phys. 85: 7472-7479; 1986 December.

[69] g, G. S.M. The molecular theory of polyelectrolyte solutions with applications to the electrostatic properties of polynucleotides; pages 179-246. 1978.

[70] Rau, D.C.; Charney, E. Polarizability of DNA. Biophys. Chem. 14: 1-9; 1981.

[71] Elias, J.G.; Eden, D. Polarizability and saline concentration. Macromolecules 14: $854-865 ; 1981$.

[72] Fixman, M. Charged macromolecules in external fields. Macromolecules 13: 711$716 ; 1980$. 
[73] Minakata, A.; Imai, N.; Oosawa, F. Dielectric properties of polyelectrolytes II. a theory of dielectric increment due to ion fluctuation by a matrix method. Biopolymers 11: 347-359; 1972.

[74] Bakhshi, A.K. Investigation of electronic conduction in proteins and DNA. Prog. Biophys. Molec. Biol. 61: 187-253; 1994.

[75] Risser, S.M.; Beratan, D.N. Electron-transfer in DNA: Predictions of exponential growth and decay coupling with donor-acceptor distance. J. Am. Chem Soc. 115: 2508-2510; 1993.

[76] Stemp, E.; Arkin, M.R.; Barton, J.K. Electron transfer between metallointercalators bound to DNA: Spectral identification of the transient intermediate. J. Am. Chem. Soc. 117: 2375; 1995.

[77] Beratan, D.N. Electron transfer through rigid molecular bridges. J. Amer. Chem. Soc. 108: 4321-4326; 1986.

[78] Dee, D.; Baur, M.E. Charge and excitation migration in DNA chains. J. Chem. Phys. 60: 541-559; 1974.

[79] Priyadarshy, S.; Risser, S.M.; Beratan, D.N. DNA is not a molecular wire: proteinlike electron-transfer predicted for an extended $\pi$-electron system. J. Phys. Chem. 100: 17678-17682; 1996.

[80] Murphy, C.J.; Arkin, M.R.; Jenkins, Y.; Ghatlia, N.D.; Bossamann, S.H.; Turro, N.J.; Barton, J.K. Long-range photoinduced electron transfer through a DNA helix. Science 262: 1025-1029; 1993.

[81] Williams, R.J. Electron transfer in biology. Molecular Phys. 68: 1-23; 1989.

[82] Hanlon, S.; Wong, L.; Pack, G.R. Proton equilibria in the minor groove of DNA. Biophys. J. 72: 291-300; 1996.

[83] Blank, M.; Goodman, R. Do electromagnetic fields interact directly with DNA? Bioelectromagn. 18: 111-115; 1997.

[84] Morgan, H.; Pethig, R. Protonic and ionic conduction in lysozyme. Chem. Soc. Faraday Trans. 1 82: 143-156; 1986. 
[85] Schwan, H.P. Linear and nonlinear electrode polarization and biological materials. Ann. Biomed. Eng. 20: 269-288; 1992.

[86] Dissado, L.A.; Hill, R.M. Anomalous low frequency dispersion. Chem. Soc. Faraday Trans. 2 80: 291-318; 1984.

[87] Pedone, F.; Bonincontro, A. Temperature dependence of DNA dielectric dispersion at radio frequency. Biochem. Biophys. Acta 1073: 58-584; 1991.

[88] Grant, E.H.; Sheppard, R.J.; South, G.P. Dielectric behavior of biological molecules in solution: Clarendon Press. 1978.

[89] Saif, B.; Mohr, R.K.; Montrose, C.J.; Litovitz, T.A. On the mechanism of dielectric behavior in aqueous DNA solutions. Biopolymers 31: 1171-1180; 1991.

[90] Takashima, S.; Gabriel, G.; Sheppard, R.J.; Grant, E.H. Dielectric behavior in DNA solution at radio and microwave frequencies (at 20 c). Biophys. J. 46: 29-34; 1984.

[91] Foster, K.R.; Epstein, B.R.; Gealt, M.A. Resonances in the dielectric absorption of DNA. Biophys. J. 52: 421-425; 1987.

[92] Gabriel, C.; Grant, E.H.; Tata, R.; Brown, P.R.; Gestblom, B.; Noreland, E. Microwave absorption in aqueous solutions of DNA. Nature 328: 145-146; 1987.

[93] Hayakawa, R.; Kanda, H.; Sakamoto, M.; Wada, Y. New apparatus for measuring the complex dielectric constant of a highly conductive material. Japanese J. Appl. Phys. 14(12): 2039-2052; 1975.

[94] Yamamoto, T.; Yamamoto, Y. Med. Bio. Eng. 14: 151; 1976.

[95] Schwan, H.P. Electrical Properties of Tissue and Cell Suspensions; chapter2, pages 147-209. Academic Press; 1957.

[96] Bernengo, J.C.; Hanss, M. Four-electrode, very-low-frequency impedance comparator for ionic solutions. Rev. Sci. Instrum. 47: 505-508; 1976.

[97] Martinsen, O.G.; Grimmes, S.; Karlsen, J. Low frequency dielectric dispersion of microporous membranes in electrolyte solution. Journal of Colloid and Interface Science 199: 107-110; 1998. 
[98] Baker-Jarvis, J.; Janezic, M.D.; Stafford, R. Analysis of an open-circuited sample holder for dielectric and magnetic measurements of liquids and powders. Natl. Inst. Stand. Technol. Tech. Note 5001; 1992.

[99] Bussey, H.E. Dielectric measurements in a shielded open circuit coaxial line. IEEE Trans. Instrum. Meas. IM-29: 120-124; 1980 June.

[100] Somlo, P.I. The discontinuity capacitance and the effective position of a shielded open circuit in a coaxial line. Proc. Inst. Radio Elec. Eng. Australia 28(1): 7-9; 1967 January.

[101] Hippel, V. Dielectric Materials and Applications. Cambridge, MA: M.I.T. Press; 1954.

[102] Scott, W.R.; Smith, G.S. Error analysis for dielectric spectroscopy using shielded open-circuited coaxial lines of general length. IEEE Trans. Instrum. Meas. IM-35: 130-137; 1986 June.

[103] Hill, P.N.; Green, H.E. In situ measurement of soil permittivity and permeability. J. Elect. Elect. Eng., Australia 2(4): 205-209; 1982 December.

[104] Jesch, R.L. Dielectric measurements of oil shales as functions of temperature and frequency. IEEE Trans. Geosci. Remote Sensing GE-22(2): 99-105; 1984 March.

[105] Stuchly, M.A.; Stuchly, S.S. Coaxial line reflection methods for measuring dielectric properties of biological substances at radio and microwave frequencies- $\mathrm{A}$ review. IEEE Trans. Instrum. Meas. IM-29: 176-183; 1980.

[106] Georgakilas, A.G.; Haveles, K.S.; Sideris, E.G. Dielectric study of the double helix to single coil transition of DNA. IEEE Trans. Dielec. Insul. 5: 26-32; 1998. 



\section{Periodical}

Journal of Research of the National Institute of Standards and Technology-Reports NIST research and development in those disciplines of the physical and engineering sciences in which the Institute is active. These include physics, chemistry, engineering, mathematics, and computer sciences. Papers cover a broad range of subjects, with major emphasis on measurement methodology and the basic technology underlying standardization. Also included from time to time are survey articles on topics closely related to the Institute's technical and scientific programs. Issued six times a year.

\section{Nonperiodicals}

Monographs-Major contributions to the technical literature on various subjects related to the Institute's scientific and technical activities.

Handbooks-Recommended codes of engineering and industrial practice (including safety codes) developed in cooperation with interested industries, professional organizations, and regulatory bodies.

Special Publications-Include proceedings of conferences sponsored by NIST, NIST annual reports, and other special publications appropriate to this grouping such as wall charts, pocket cards, and bibliographies.

Applied Mathematics Series-Mathematical tables, manuals, and studies of special interest to physicists, engineers, chemists, biologists, mathematicians, computer programmers, and others engaged in scientific and technical work.

National Standard Reference Data Series-Provides quantitative data on the physical and chemical properties of materials, compiled from the world's literature and critically evaluated. Developed under a worldwide program coordinated by NIST under the authority of the National Standard Data Act (Public Law 90-396). NOTE: The Journal of Physical and Chemical Reference Data (JPCRD) is published bimonthly for NIST by the American Chemical Society (ACS) and the American Institute of Physics (AIP). Subscriptions, reprints, and supplements are available from ACS, 1155 Sixteenth St., NW, Washington, DC 20056.

Building Science Series-Disseminates technical information developed at the Institute on building materials, components, systems, and whole structures. The series presents research results, test methods, and performance criteria related to the structural and environmental functions and the durability and safety characteristics of building elements and systems.

Technical Notes-Studies or reports which are complete in themselves but restrictive in their treatment of a subject. Analogous to monographs but not so comprehensive in scope or definitive in treatment of the subject area. Often serve as a vehicle for final reports of work performed at NIST under the sponsorship of other government agencies.

Voluntary Product Standards-Developed under procedures published by the Department of Commerce in Part 10, Title 15, of the Code of Federal Regulations. The standards establish nationally recognized requirements for products, and provide all concerned interests with a basis for common understanding of the characteristics of the products. NIST administers this program in support of the efforts of privatesector standardizing organizations.

Consumer Information Series-Practical information, based on NIST research and experience, covering areas of interest to the consumer. Easily understandable language and illustrations provide useful background knowledge for shopping in today's technological marketplace.

Order the above NIST publications from: Superintendent of Documents, Government Printing Office, Washington, DC 20402.

Order the following NIST publications-FIPS and NISTIRs-from the National Technical Information Service, Springfield, VA 22161.

Federal Information Processing Standards Publications (FIPS PUB)-Publications in this series collectively constitute the Federal Information Processing Standards Register. The Register serves as the official source of information in the Federal Government regarding standards issued by NIST pursuant to the Federal Property and Administrative Services Act of 1949 as amended, Public Law 89-306 (79 Stat. 1127), and as implemented by Executive Order 11717 (38 FR 12315, dated May 11, 1973) and Part 6 of Title 15 CFR (Code of Federal Regulations).

NIST Interagency Reports (NISTIR)-A special series of interim or final reports on work performed by NIST for outside sponsors (both government and non-government). In general, initial distribution is handled by the sponsor; public distribution is by the National Technical Information Service. Springfield. VA 22161, in paper copy or microfiche form. 
U.S. Department of Commerce

National Institute of Standards and Technology

325 Broadway

Boulder, Colorado 80303-3328

Official Business

Penalty for Private Use, $\$ 300$ 